\title{
Droplet Evaporation on Hot Micro-Structured Superhydrophobic Surfaces: Analysis of Evaporation from Droplet Cap and Base Surfaces
}

\author{
Wenge Huang ${ }^{\mathrm{a}, 1}$, Xukun He ${ }^{\mathrm{a}, 1}$, Cong Liu ${ }^{\mathrm{b}}$, Xiaojie Li ${ }^{\mathrm{b}}$, Yahua Liu ${ }^{\mathrm{b}}$, C. Patrick Collier ${ }^{\mathrm{c}}$, \\ Bernadeta R. Srijanto ${ }^{c}$, Jiansheng Liu ${ }^{d}$, Jiangtao Cheng ${ }^{a, *}$ \\ a Department of Mechanical Engineering, Virginia Tech, Blacksburg, VA 24061, USA \\ ${ }^{\mathrm{b}}$ Key Laboratory for Non-Traditional Machining Technology of Ministry of Education, Dalian University of Technology, Dalian 116024, China \\ c Center for Nanophase Materials Sciences, Oak Ridge National Laboratory, Oak Ridge, TN 37831, USA \\ ${ }^{\mathrm{d} S c h o o l}$ of Electronic and Information Engineering, Beihang University, 37 Xueyuan Road, Beijing 100191, China
}

\section{A R T I C L E I N F O}

\section{Article history:}

Received 19 July 2021

Revised 22 November 2021

Accepted 23 November 2021

Available online 4 December 2021

\section{Keywords:}

droplet evaporation

micro-structured surfaces

superhydrophobic surfaces

thermal circuit analysis

depressed boiling

evaporative cooling

\begin{abstract}
A B S T R A C T
In this study, evaporation of sessile water droplets on hot micro-structured superhydrophobic surfaces is experimentally and theoretically investigated. Water droplets of $4 \mu \mathrm{L}$ are placed on micro-pillared silicon substrates with the substrate temperature heated up to $120^{\circ} \mathrm{C}$. A comprehensive thermal circuit model is developed to analyze the effects of substrate roughness and substrate temperature on the sessile droplet evaporation. For the first time, two components of heat and mass transfer, i.e., one from the droplet cap surface and the other from the droplet base surface, during droplet evaporation are distinguished and systematically studied. As such, the evaporation heat transfer rates from both the droplet cap surface and the interstitial liquid-vapor interface between micropillars at the droplet base are calculated in various conditions. For droplet evaporation on the heated substrates in the range of $40^{\circ} \mathrm{C}-80^{\circ} \mathrm{C}$, the predicted droplet cap temperature matches well with the experimental results. During the constant contact radius mode of droplet evaporation, the decrease of evaporation rate from the droplet base contributes most to the continuously decreasing overall evaporation heat transfer rate, whereas the decrease of evaporation rate from the droplet cap surface is dominant in the constant contact angle mode. The influence of internal fluid flow is considered for droplet evaporation on substrates heated above $100^{\circ} \mathrm{C}$, and an effective thermal conductivity is adopted as a correction factor to account for the effect of convection heat transfer inside the droplet. Temperature differences between the droplet base and the substrate base are estimated to be about $2^{\circ} \mathrm{C}, 5^{\circ} \mathrm{C}, 8^{\circ} \mathrm{C}, 13^{\circ} \mathrm{C}$ and $18^{\circ} \mathrm{C}$ for droplet evaporation on substrates heated at $40^{\circ} \mathrm{C}$, $60^{\circ} \mathrm{C}, 80^{\circ} \mathrm{C}, 100^{\circ} \mathrm{C}$, and $120^{\circ} \mathrm{C}$, respectively, elucidating the delayed or depressed boiling of water droplets on a heated rough surface due to evaporative cooling.
\end{abstract}

(c) 2021 Elsevier Ltd. All rights reserved.

\section{Introduction}

Evaporation of sessile liquid droplets is a ubiquitous phenomenon in nature, which plays an important role in a variety of applications, including inkjet printing [1], DNA mapping [2], spray cooling [3], analyte enrichment/detection [4], and colloidal assembly [5]. Yet, sessile droplet evaporation is a complex process controlled by several interdependent factors, such as droplet contact angle dynamics [6], contact line motion [7,8], substrate structures

\footnotetext{
* Corresponding author.

E-mail address: chengjt@vt.edu (J. Cheng).

1 Wenge Huang and Xukun He are co-first authors with equal contribution to this work.
}

and temperature $[9,10]$, and the surrounding environment [11,12], which deserve further investigation.

Since the seminal work of Picknett and Bexon in 1977 [13], sessile droplet evaporation on smooth surfaces has been systematically studied and categorized with one of the three evaporation modes: (1) constant contact radius (CCR) mode: the droplet contact line is pinned with a constant contact radius while the contact angle keeps decreasing; (2) constant contact angle (CCA) mode: once the contact angle approaches the receding contact angle, the contact line keeps receding with the contact angle unchanged; (3) mixed mode: both the contact radius and contact angle decrease near the end of evaporation [14]. In several recent studies about droplet evaporation on structured superhydrophobic surfaces, a special stick-slip behavior [15] was observed, in which 


\begin{tabular}{|c|c|}
\hline \multicolumn{2}{|c|}{ Nomenclature } \\
\hline$a$ & radius of micropillar $[\mu \mathrm{m}]$ \\
\hline$b$ & $\begin{array}{l}\text { radius of water/substrate layer in one unit cell } \\
{[\mu \mathrm{m}]}\end{array}$ \\
\hline$c_{\mathrm{Si}}$ & specific heat capacity of silicon $[\mathrm{J} /(\mathrm{kg} \cdot \mathrm{K})]$ \\
\hline$c_{\mathrm{S}}$ & saturated vapor concentration $\left[\mathrm{kg} / \mathrm{m}^{3}\right]$ \\
\hline$c_{\mathrm{W}}$ & specific heat capacity of water $[\mathrm{J} /(\mathrm{kg} \cdot \mathrm{K})]$ \\
\hline$D$ & diameter of micropillar $[\mu \mathrm{m}]$ \\
\hline$D_{\mathrm{d}}$ & coefficient of vapor diffusion $\left[\mathrm{m}^{2} / \mathrm{s}\right]$ \\
\hline$D_{\max }$ & the maximum diameter of the droplet cap [m] \\
\hline e & coefficient of effective conductivity \\
\hline$H$ & height of micropillar $[\mu \mathrm{m}]$ \\
\hline$h_{\text {conv }}$ & $\begin{array}{l}\text { convection heat transfer coefficient of air } \\
{\left[\mathrm{W} /\left(\mathrm{m}^{2} \cdot \mathrm{K}\right)\right]}\end{array}$ \\
\hline$h_{\mathrm{fg}}$ & latent heat of water $[\mathrm{kJ} / \mathrm{kg}]$ \\
\hline$J_{0}(x)$ & the first kind Bessel function with order of 0 \\
\hline$J_{1}(x)$ & the first kind Bessel function with order of 1 \\
\hline$J_{m}$ & local evaporation mass flux $\left[\mathrm{kg} /\left(\mathrm{m}^{2} \cdot \mathrm{s}\right)\right]$ \\
\hline$k_{\mathrm{eff}, \mathrm{w}}$ & effective conductivity of water $[\mathrm{W} /(\mathrm{m} \cdot \mathrm{K})]$ \\
\hline$k_{\mathrm{Si}}$ & thermal conductivity of silicon $[\mathrm{W} /(\mathrm{m} \cdot \mathrm{K})]$ \\
\hline$k_{\mathrm{w}}$ & thermal conductivity of water $[\mathrm{W} /(\mathrm{m} \cdot \mathrm{K})]$ \\
\hline$l_{\mathrm{s}}$ & thickness of silicon substrate $[\mu \mathrm{m}]$ \\
\hline$l_{\mathrm{w}}$ & thickness of water layer $[\mu \mathrm{m}]$ \\
\hline$M$ & $\begin{array}{l}\text { number of water layers in the upper hemisphere } \\
\text { of the droplet spherical cap }\end{array}$ \\
\hline$N$ & number of micropillars under the droplet base \\
\hline$n$ & order of Bessel function \\
\hline$P$ & periodicity of substrate $[\mu \mathrm{m}]$ \\
\hline$P_{-0.5+i \omega}(x)$ & the first kind Legendre function \\
\hline$q_{b}$ & $\begin{array}{l}\text { heat transfer rate from liquid-vapor interface at } \\
\text { droplet base in one unit cell [W] }\end{array}$ \\
\hline$q_{\text {conv }}$ & convective heat transfer rate [W] \\
\hline$q_{\mathrm{c}}$ & $\begin{array}{l}\text { evaporation heat transfer rate from droplet cap } \\
\text { surface [W] }\end{array}$ \\
\hline$q_{\text {evap }}$ & $\begin{array}{l}\text { evaporation heat transfer rate of water droplet } \\
\text { [W] }\end{array}$ \\
\hline$q_{1, \mathrm{i}}$ & $\begin{array}{l}\text { heat transfer rate from the side surface water } \\
\text { layer [W] }\end{array}$ \\
\hline$q_{\mathrm{p}}$ & $\begin{array}{l}\text { heat transfer rate from substrate to droplet in } \\
\text { one unit cell [W] }\end{array}$ \\
\hline$q_{\mathrm{rad}}$ & radiative heat transfer rate $[\mathrm{W}]$ \\
\hline$q_{\text {rise }}$ & $\begin{array}{l}\text { energy transfer rate for bulk water temperature } \\
\text { increase }[\mathrm{W}]\end{array}$ \\
\hline$q_{\mathrm{s}}$ & $\begin{array}{l}\text { heat transfer rate from substrate to droplet base } \\
\text { [W] }\end{array}$ \\
\hline$q_{\mathrm{w}}$ & $\begin{array}{l}\text { heat transfer rate in the water layer in one unit } \\
\text { cell }[\mathrm{W}]\end{array}$ \\
\hline$R_{\mathrm{h}}$ & relative humidity \\
\hline$R_{\mathrm{i}}$ & thermal resistance of water layer $[\mathrm{K} / \mathrm{W}]$ \\
\hline$R_{\mathrm{S}}$ & thermal resistance of substrate $[\mathrm{K} / \mathrm{W}]$ \\
\hline$r$ & radius of water droplet $[\mu \mathrm{m}]$ \\
\hline$r_{\mathrm{w}}$ & radius of spherical cap droplet $[\mu \mathrm{m}]$ \\
\hline$S$ & surface of spherical cap $\left[\mu \mathrm{m}^{2}\right]$ \\
\hline $\bar{T}_{\mathrm{b}}$ & average temperature of droplet base $[\mathrm{K}]$ \\
\hline$T_{\mathrm{b}, \mathrm{p}}$ & average temperature of solid-liquid interface $[\mathrm{K}]$ \\
\hline$T_{\text {bulk }}$ & droplet bulk temperature $[\mathrm{K}]$ \\
\hline$T_{\text {cap }}$ & temperature of droplet cap surface $[\mathrm{K}]$ \\
\hline$T_{\mathrm{e}}$ & $\begin{array}{l}\text { contact temperature at the solid-liquid interface } \\
{[\mathrm{K}]}\end{array}$ \\
\hline$T_{\text {expt }}$ & $\begin{array}{l}\text { droplet surface temperature tested by the IR } \\
\text { camera }\end{array}$ \\
\hline$T_{\mathrm{i}}$ & temperature of control volume water layer [K] \\
\hline$T_{\text {lab }}$ & lab ambient temperature $[\mathrm{K}]$ \\
\hline
\end{tabular}

\begin{tabular}{|c|c|}
\hline$T_{\text {model }}$ & $\begin{array}{l}\text { average temperature calculated by the thermal } \\
\text { circuit model }[\mathrm{K}]\end{array}$ \\
\hline$T_{0}$ & initial temperature of droplet base $[\mathrm{K}]$ \\
\hline$T_{\mathrm{p}}$ & top surface temperature of micropillar $[\mathrm{K}]$ \\
\hline$T_{\mathrm{w}}$ & $\begin{array}{l}\text { uniform temperature of water layer in one unit } \\
\text { cell }[\mathrm{K}]\end{array}$ \\
\hline$T_{\infty}$ & ambient temperature $[\mathrm{K}]$ \\
\hline$V$ & volume of water droplet \\
\hline$X$ & total number of water layers \\
\hline \multicolumn{2}{|c|}{ Greek symbols } \\
\hline$\alpha$ & root of Bessel function \\
\hline$\beta$ & toroidal coordinate \\
\hline$\varepsilon$ & emissivity of water \\
\hline$\theta$ & contact angle of water droplet $\left[{ }^{\circ}\right]$ \\
\hline$\rho$ & density $\left[\mathrm{kg} / \mathrm{m}^{3}\right]$ \\
\hline$\sigma$ & Stefan-Boltzmann constant $\left[\mathrm{kg} /\left(\mathrm{s}^{3} \cdot \mathrm{K}^{4}\right)\right]$ \\
\hline$T$ & nondimensional evaporation time \\
\hline$\Omega$ & the variable of integration \\
\hline$\varphi$ & evaporation ratio \\
\hline \multicolumn{2}{|c|}{ Abbreviations } \\
\hline CCA & constant contact angle \\
\hline CCR & constant contact radius \\
\hline DI & deionized \\
\hline IR & infrared \\
\hline SEM & scanning electron microscope \\
\hline
\end{tabular}

the droplet contact line is moved by the pinning and depinning forces alternately.

Several theoretical models have been proposed to predict the evaporation rate of sessile droplets on various surfaces. In the classical work of Picknett and Bexon [13], the sessile droplet evaporation at room temperature was assumed to be primarily driven by vapor diffusion, ignoring the convection heat transfer inside and outside the droplet. And the analytic evaporation rate could be obtained based on an analogy between the concentration field and the electrostatic field [16]. During the past two decades, this diffusion-driven model has been widely applied in modeling the evaporation of sessile droplets with an arbitrary contact angle in the CCR mode, or with a slipping contact line in the CCA mode. The excellent agreement between the analytical evaporation rate and the experimental data confirms the validity of the diffusion-driven model of sessile droplet evaporation, not only on hydrophilic surfaces [17-19] but also on hydrophobic surfaces [20,21].

However, when the diffusion-driven model was employed for analyzing sessile droplet evaporation on non-wetting surfaces with microstructures [22], especially on heated superhydrophobic surfaces, an overestimation of the evaporation rate was observed by Garimalla [23,24], Aldhaleai [25] and Bussonniere [26]. This deviation from the analytically predicted evaporation rate should result from evaporative cooling, giving rise to a temperature reduction on the liquid-vapor interface, which is in contradiction to the pivotal assumption made in the classical diffusion-driven model that the temperature of the droplet surface is constant and the same as the substrate temperature [17-19]. For instance, the maximum temperature mismatch of $\sim 20^{\circ} \mathrm{C}$ between the droplet surface and the substrate was experimentally observed when the substrate was heated at $70^{\circ} \mathrm{C}$ [27]. Furthermore, the applicability of the diffusion-driven model might become even worse when the wetting states of a droplet on micro-structured surfaces, i.e., the Cassie state or the Wenzel state [28], are considered. For the evaporation of a sessile droplet in the Cassie state, the existence of the air/vapor cushion layer between the droplet base and the microstructures would lead 
to two different components of droplet evaporation, i.e., one from the liquid-vapor interface at the droplet cap and the other from the droplet base. However, the effect of this extra evaporative interface at the droplet base on superhydrophobic surfaces was generally disregarded in the majority of previous work [23,24,29]. Indeed, in recent studies of Wang [30,31] and Kim [32], the non-negligible evaporation flux through the vapor-liquid interface over the air pockets has been experimentally confirmed on heated superhydrophobic surfaces, but these work mainly focused on the wetting or dynamics of the evaporating droplet. Therefore, a systematic study about droplet evaporation on heated micro-structured surfaces by considering the comprehensive effects of both the discontinuous liquid-vapor interfaces at the droplet base and the continuous liquid-vapor interface at the droplet cap is entailed.

In this paper, the evaporation of water droplets on hot microstructured superhydrophobic substrates is experimentally and theoretically investigated. First, a water microdroplet was placed on the superhydrophobic substrates heated at $40^{\circ} \mathrm{C}-80^{\circ} \mathrm{C}$. The droplet evaporated in the Cassie state during most of its evaporation time, and then transited to the Wenzel state at the very end of evaporation. Based on a comprehensive thermal resistance analysis, a thermal circuit model was developed to predict the droplet cap surface temperature and to calculate the evaporation heat transfer rates from the droplet cap surface and the base surface, respectively. Then, the substrate was further heated from $80^{\circ} \mathrm{C}$ to $120^{\circ} \mathrm{C}$ until an additional small increase of the substrate temperature would otherwise lead to the boiling of the droplet. As such, an effective thermal conductivity was adopted as a correction factor to account for the effect of convection heat transfer inside the water droplet. The average temperature of the droplet base surface was calculated and then the temperature difference between the droplet base and the substrate base was obtained, manifested as the depressed or delayed boiling of droplets on the superheated substrates. This study could deepen our understanding of droplet evaporation on hot micro-structured surfaces and potentially provide us better control of sessile droplet evaporation on non-wetting surfaces.

\section{Experimental methodology}

\subsection{Substrates with micropillars}

Micropillared silicon substrates were fabricated by standard contact photolithography process and deep reactive ion etching method [4]. Three kinds of substrates of $500 \mu \mathrm{m}$ thick and textured with cylindrical micropillar arrays with varying micropillar periodicity $(P)$ were fabricated in this study. The geometry information of the sample substrates is listed in Table 1 and the scanning electron microscope (SEM) image of one sample substrate is shown in Fig. 1(a). All the substrates were conformally coated with silane (Trichloro $(1 \mathrm{H}, 1 \mathrm{H}, 2 \mathrm{H}, 2 \mathrm{H}$-per fluorooctyl)-silane, Sigma-Aldrich) using standard chemical vapor deposition process for superhydrophobicity enhancement [33]. Then the substrates were baked on a hot plate at $100^{\circ} \mathrm{C}$ for $60 \mathrm{~min}$. Water droplet exhibits a contact angle of $155^{\circ} \pm 2^{\circ}$ on thus-prepared substrates. To mitigate the sample edge effect on the droplet evaporation process, substrate samples were cut into square pieces with the dimension of $2 \mathrm{~cm} \times 2 \mathrm{~cm}$

Table 1

Micropillar diameter, periodicity and height on the sample substrates.

\begin{tabular}{llll}
\hline Substrate & $\begin{array}{l}\text { Micropillar } \\
\text { diameter D }(\mu \mathrm{m})\end{array}$ & $\begin{array}{l}\text { Micropillar } \\
\text { periodicity P }(\mu \mathrm{m})\end{array}$ & $\begin{array}{l}\text { Micropillar } \\
\text { height } \mathrm{H}(\mu \mathrm{m})\end{array}$ \\
\hline Sample 1 & 20 & 40 & 40 \\
Sample 2 & 20 & 50 & 40 \\
Sample 3 & 20 & 60 & 40 \\
\hline
\end{tabular}

and water droplets were deposited at the center of each piece for evaporation study.

\subsection{Experimental setup}

The schematic of the experimental setup is shown in Fig. 1(b). In this study, deionized (DI) water (Type $1,>18 \mathrm{M} \Omega \mathrm{cm}$ resistivity) was used as the liquid. A DI water droplet of $4 \pm 0.1 \mu \mathrm{L}$ was generated by a syringe pump (EW-74905, Cole-Parmer) through a FISNAR dispense tip with $0.15 \mathrm{~mm}$ internal diameter and $0.30 \mathrm{~mm}$ external diameter. Then the water droplet was gently dispensed on the center of the micro-structured substrate for evaporation study. At least five trials were carried out for each droplet evaporation experiment to ensure the repeatability of the evaporation measurements. Because of the small volume, the shape of the sessile water droplet resembled a spherical cap. The substrate was intimately affixed on a hot plate by double-side copper tape and a K-type thermocouple with $\pm 0.5^{\circ} \mathrm{C}$ uncertainty was used to measure the surface temperature of the substrate during the experiment. The base temperature of the substrate was maintained at a constant level on the hot plate ranging from $40^{\circ} \mathrm{C}$ to $120^{\circ} \mathrm{C}$, beyond which even a small temperature increase would otherwise lead to the onset of boiling of the sessile droplet. Two cameras parallel and normal to the substrate were used to record the evaporation process of the droplets. An infrared (IR) camera (FLIR A655sc) was fixed above the droplet and normal to the substrate to measure the surface temperature evolution of the droplet cap. The detailed calibration process of the IR camera is given in the supplementary materials. A water droplet sitting on the superhydrophobic substrate exhibits a large contact angle and only the upper hemispherical surface of the droplet cap can be focused by the IR camera. Regardless of the influence of droplet internal flow on its surface temperature distribution, the temperature measured by the IR camera was taken as the average surface temperature of the upper hemispherical cap of the droplet. Aligned in parallel to the substrate, a high-speed CCD camera mated with an optical tensiometer (Theta Lite, OneAttension Corporation) was used to capture the images of the evaporating droplet. The droplet shape was assumed to be axisymmetric. Based on the captured snapshots, the water droplet was vertically divided into multiple layers and the local height and diameter of each water layer were obtained by image processing. Then, the droplet volume was calculated by integrating the volume of each discrete layer. With the snapshots obtained by the high-speed camera, the transient droplet volume, contact angle, contact radius and droplet height were collected for subsequent analysis. During the experiment, the ambient temperature and relative humidity were maintained at $T_{\mathrm{lab}}=23 \pm 2^{\circ} \mathrm{C}$ and $R_{\mathrm{h}}=35 \pm 5 \%$, respectively.

\section{Analytical methodology}

\subsection{Wetting state}

Water droplets can exhibit different wetting states on the micro-structured substrate during the evaporation. Specific air/vapor pockets underneath the droplet were observed during the majority period of the evaporation, indicating that the droplet was at least partially in the Cassie state. At the very end of the evaporation, water was observed to completely fill the interstitial spacing underneath the droplet and the droplet was in the Wenzel state during this period. The snapshots of the water droplet during evaporation are shown in Fig. 2. Though the droplet exhibited two distinct wetting states during evaporation, the droplet stayed in the Cassie state during most of the evaporation process. In this work, we focus on the evaporation process of the droplet in the Cassie state and the thermal circuit model developed by us is based on the Cassie droplet. 
(a)

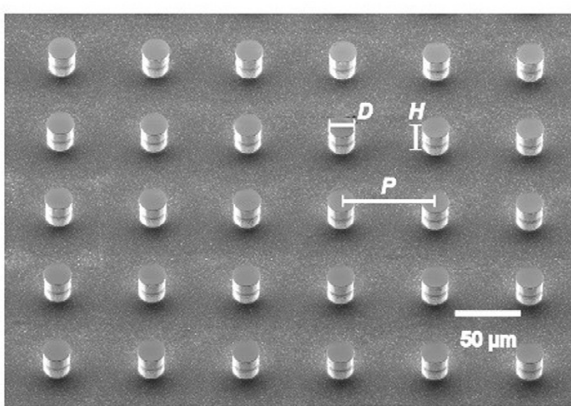

(b)

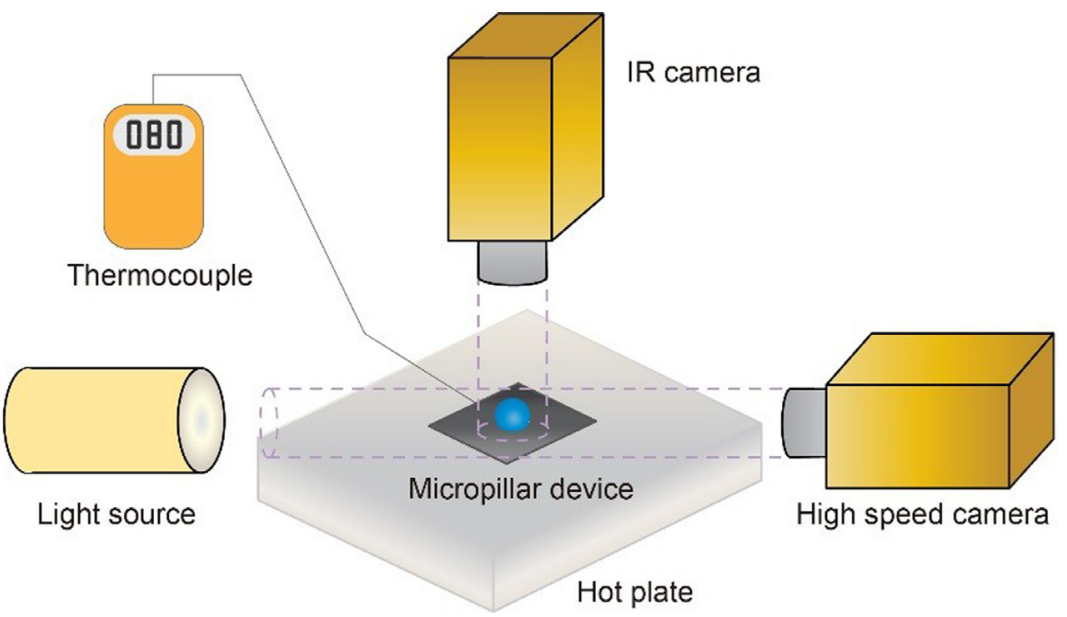

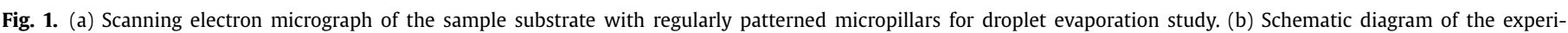

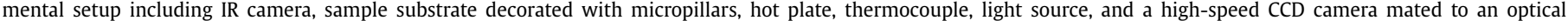
tensiometer.

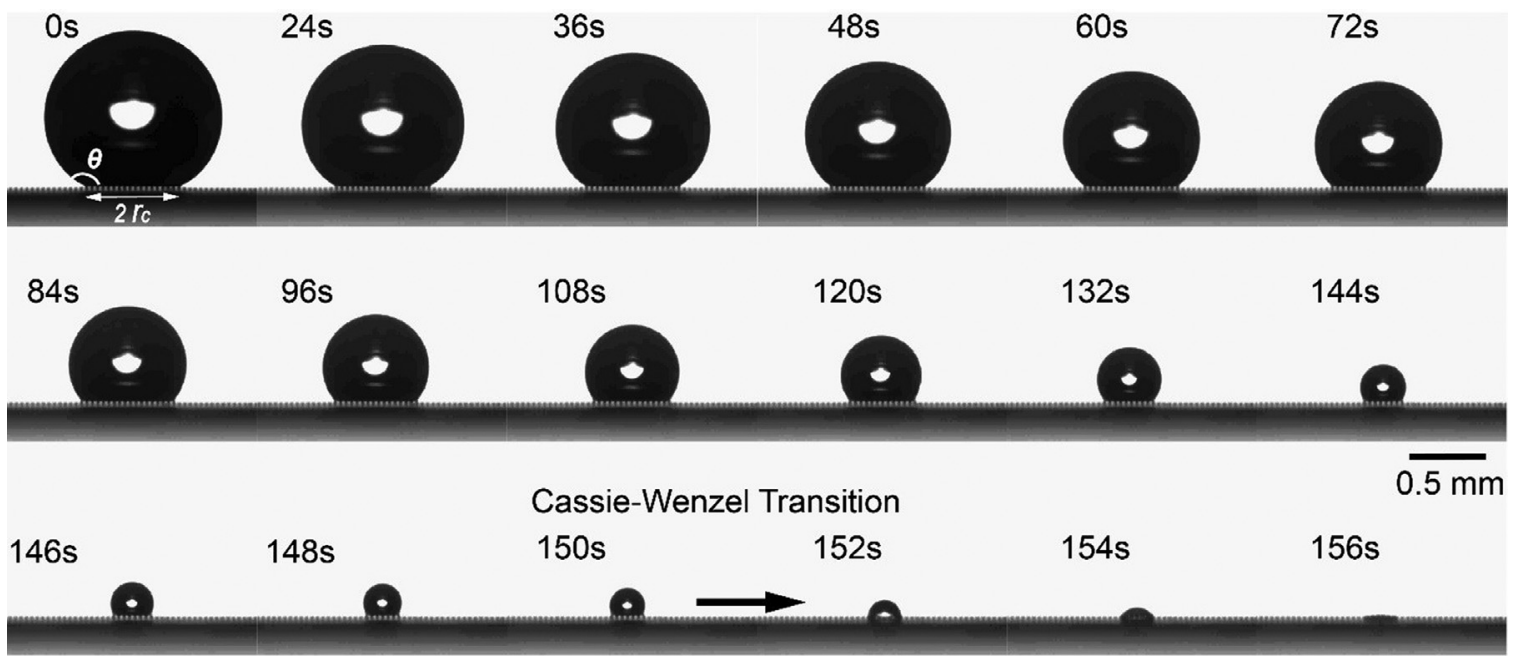

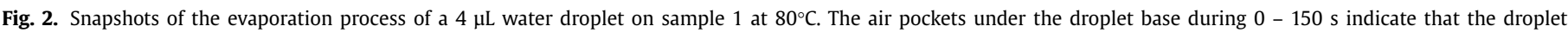

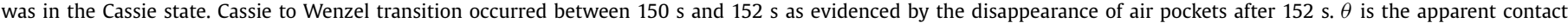
angle of droplet on a rough surface.

\subsection{Energy balance model}

When a droplet is deposited on the hot substrate surface, heat transfers from the hot substrate through the micropillars into the sessile droplet due to the temperature difference between them as shown in Fig. 3(a). This heat transfer process results in the temperature increase inside the droplet and accelerate the heat and mass transfer between the droplet and the ambient air. The energy balance of the evaporating sessile droplet is given by:

$q_{\mathrm{s}}=q_{\text {rise }}+q_{\text {conv }}+q_{\text {rad }}+q_{\text {evap }}$

where $q_{\mathrm{s}}$ is the overall heat transfer rate from the substrate to the droplet, $q_{\text {rise }}$ accounts for the sensible heat required for the temperature increase within the bulk water, $q_{\text {conv }}$ is the convective heat transfer rate between the water droplet and the ambient, $q_{\text {rad }}$ is the radiation heat transfer rate from the droplet surface to the ambient, and $q_{\text {evap }}$ is the evaporation heat transfer rate. So, we have:

$q_{\text {rise }}=c_{\mathrm{w}} \rho_{\mathrm{w}} V \frac{d T_{\text {bulk }}}{d t}$

$q_{\mathrm{rad}}=\varepsilon \sigma S\left(T_{\mathrm{cap}}^{4}-T_{\infty}^{4}\right)$ $q_{\mathrm{conv}}=h_{\mathrm{conv}} S\left(T_{\mathrm{cap}}-T_{\infty}\right)$

$q_{\text {evap }}=h_{\mathrm{fg}} \rho_{\mathrm{w}} \frac{d V}{d t}$

where $c_{\mathrm{w}}, \rho_{\mathrm{w}}, T_{\text {cap }}, T_{\text {bulk }}, V$ and $h_{\mathrm{fg}}$ are the specific heat capacity, density, droplet cap surface temperature, droplet bulk temperature, volume and latent heat of the water droplet, respectively; $\varepsilon$ is the emissivity of the water droplet surface and $\sigma$ is the StefanBoltzmann constant; $S$ is the liquid-vapor interface area and $h_{\text {conv }}$ is the natural convection heat transfer coefficient of the ambient air.

Because of the high efficiency of phase change heat transfer, the evaporation heat transfer rate $q_{\text {evap }}$ is dominant over the other heat transfer modes. Thus, the overall heat transfer from the substrate to the water droplet can be estimated as the heat released from the droplet surface to the ambient by evaporation [34]. Therefore, the energy balance of the sessile droplet can be rewritten as:

$q_{\mathrm{s}} \approx q_{\mathrm{evap}}$ 

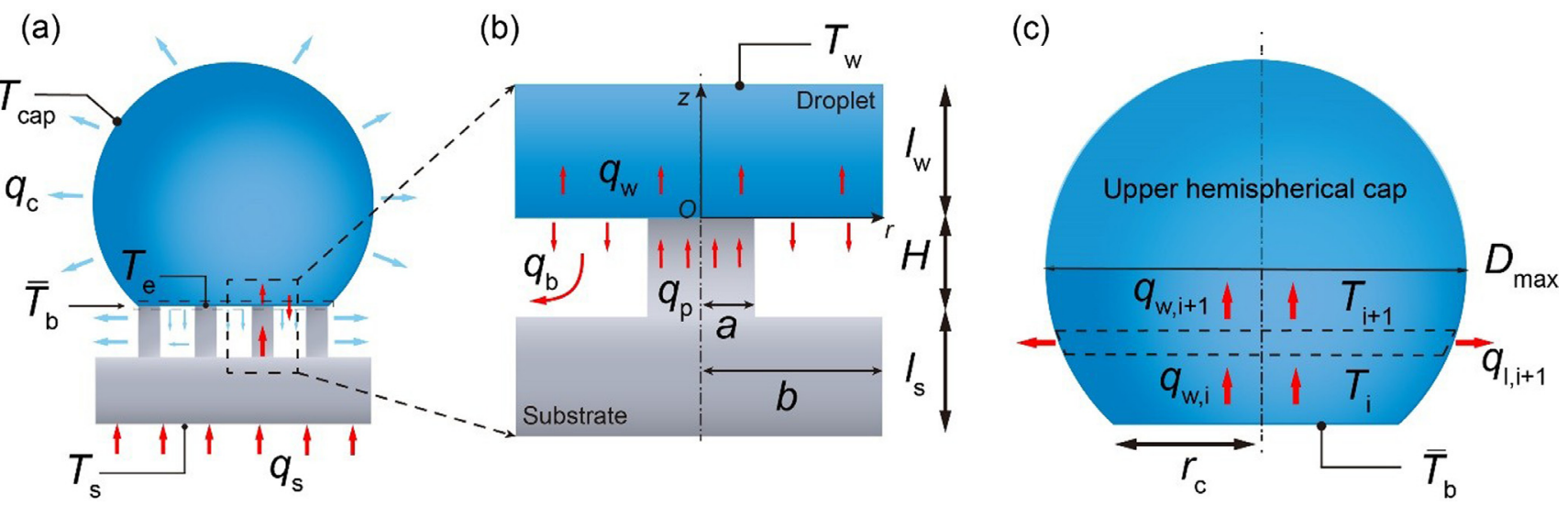

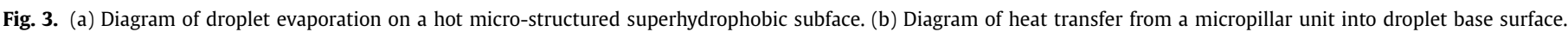

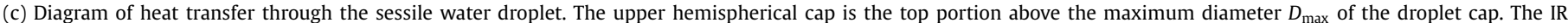
camera above a sessile droplet can only detect the temperature distribution on the upper hemispherical surface.

\subsection{Average temperature of droplet base surface}

In section 3.2, by analyzing the energy balance of a water droplet during evaporation, we found that the heat transfer rate from the substrate $q_{\mathrm{s}}$ can be estimated by the evaporative heat transfer rate $q_{\text {evap. }}$. Because of the periodicity of the micropillar array, the heat transfer process in one micropillar cell as illustrated in Fig. 3(b) can be representative of the heat transfer process between the droplet base and the substrate micropillars [30,35]. Thus, we focus on the heat transfer from one micropillar cell into the droplet base and the heat transfer rate in one unit cell is calculated as:

$q_{\mathrm{p}}=\frac{q_{\mathrm{s}}}{N}=\frac{q_{\mathrm{evap}}}{N}$

where $N$ is the total number of the micropillars underneath the droplet, i.e., the ratio of the droplet apparent contact area to one unit cell area.

A unit of the micropillar cell consists of one micropillar and the air pocket around it. In general, heat transfer from both the silicon micropillar and the vapor pocket surrounding it should be considered. However, the thermal resistance of the vapor layer is much larger than that of the silicon micropillar due to the order of magnitude difference in the thermal conductivities of silicon micropillars $\left(100 \mathrm{~W} \cdot \mathrm{m}^{-1} \cdot \mathrm{K}^{-1}\right)[30]$ and water vapor $\left(0.025 \mathrm{~W} \cdot \mathrm{m}^{-1} \cdot \mathrm{K}^{-1}\right)$ [31]. Therefore, it is reasonable to assume that heat primarily conducts from the micropillar to the water droplet while the vaporsolid interface of the air pocket can be regarded as adiabatic. The thermal resistance per unit cell of the silicon substrate can be calculated as:

$R_{\mathrm{s}}=\frac{l_{\mathrm{s}}}{k_{\mathrm{Si}} P^{2}}+\frac{4 H}{k_{\mathrm{Si}} \pi D^{2}}$

where $k_{\mathrm{Si}}$ is the thermal conductivity of silicon and $l_{\mathrm{S}}$ is the thickness of the silicon substrate, excluding the micropillar height.

The effects of droplet internal flow are not significant for droplet evaporation on a relatively low temperature substrate, i.e., $<80^{\circ} \mathrm{C}$. The characteristic fluid velocity in a $3 \mu \mathrm{L}$ water droplet evaporating on a $60^{\circ} \mathrm{C}$ substrate is about tens microns per second and the Peclet number is less than 1 [23]. Thus, it is reasonable to consider just the conduction heat transfer while neglecting the internal convection of the water droplet on a substrate with a relatively low temperature. The time scale for heat conduction in the droplet is on the order of $\frac{r_{\mathrm{w}}^{2} \rho_{\mathrm{w}} c_{\mathrm{w}}}{k_{\mathrm{w}}} \sim 0.01 \mathrm{~s}$ [36], where $r_{\mathrm{w}}$ is the radius of the droplet. The total evaporation time of the droplet on the substrate is on the order of $100 \mathrm{~s}$. The ratio of the time scale for heat conduction to that of evaporation is about 0.0001 , implying that the conductive heat transfer process may be considered as quasi steady.

To estimate the temperature distribution near the liquid-solid interface, i.e., at the tip of the micropillar in contact with the droplet base, a thin water layer with a thickness of $l_{\mathrm{w}}$ atop a unit cell (Fig. $3 \mathrm{~b}$ ) is considered. The conductive heat transfer equation for the water layer therein is:

$\frac{\partial^{2} T}{\partial r^{2}}+\frac{1}{r} \frac{\partial T}{\partial r}+\frac{\partial^{2} T}{\partial z^{2}}=0$

where $T$ denotes the temperature of the water film.

Due to the relatively small size of the micropillar, the heat flux across the liquid-solid (tip) interface and the liquid-vapor interface in a unit cell could be assumed to be uniform. As a result, we have the first boundary condition:

$k_{\mathrm{w}} \frac{\partial T}{\partial z}= \begin{cases}\frac{q_{\mathrm{p}}}{\pi a^{2}} & 0<r<a ; \quad z=0 \\ -\frac{q_{\mathrm{b}}}{\pi\left(b^{2}-a^{2}\right)} & a<r<b ; \quad z=0\end{cases}$

where $k_{w}$ is the thermal conductivity of water, $a$ is the radius of the micropillar, $b$ is the radius of one unit cell [37], $q_{\mathrm{b}}$ is the heat transfer rate from the droplet base-vapor interface within one unit cell as shown in Fig. 3(b).

Temperature inside the water layer is assumed to quickly become uniform. Hence, a uniform temperature boundary could be assumed at $z=l_{w}$ :

$T(r ; z)=T_{w} \quad 0<r<b ; \quad z=l_{w}$

Moreover, considering the periodicity of the unit cells, we assume the adiabatic boundary between the chosen unit cell and its neighboring cells inside the water layer. Thus, we obtain the following adiabatic boundary condition:

$\frac{\partial T}{\partial r}=0 \quad r=b ; \quad 0<z<l_{w}$

Solving the heat transfer equation Eq. (11) with the three boundary conditions Eqs. (10)-(12), we obtain the temperature distribution inside the water layer as:

$$
\begin{aligned}
T_{\mathrm{b}}(\mathrm{r}, \mathrm{z})= & T_{\mathrm{w}}+\frac{q_{p}\left(l_{\mathrm{w}}-z\right)}{\pi k_{\mathrm{w}}}\left(\frac{(1-\varphi)}{b^{2}}\right)+\frac{2 a q_{\mathrm{p}}}{\pi k_{\mathrm{w}}}\left(\frac{1}{a^{2}}+\frac{\varphi}{b^{2}-a^{2}}\right) \sum_{n=1}^{\infty} \\
& {\left[J_{1}\left(\frac{a}{b} \alpha_{n}\right) J_{0}\left(\frac{r}{b} \alpha_{n}\right) /\left(\alpha_{n}^{2} J_{0}^{2}\left(\alpha_{n}\right)\right)\right] \frac{\sinh \left(\frac{\alpha_{n}}{b}\left(l_{\mathrm{w}}-z\right)\right)}{\cosh \left(\frac{\alpha_{n}}{b} l_{\mathrm{w}}\right)} }
\end{aligned}
$$


where $\varphi$ is the evaporation ratio, i.e., the heat transfer across the droplet base-vapor interface over the overall heat transfer from the substrate to the droplet $\varphi=q_{\mathrm{b}} / q_{\mathrm{p}}$. Here $J_{0}(x)$ and $J_{1}(x)$ are the first kind Bessel functions with orders of 0 and 1 , respectively, $\alpha_{n}$ is the $n^{\text {th }}$ root of $J_{1}(x)=0$ [38].

The temperature at the droplet base $(z=0)$ is calculated as:

$$
\begin{aligned}
T_{\mathrm{b}}(\mathrm{r}, 0)= & T_{\mathrm{w}}+\frac{q_{p} l_{\mathrm{w}}}{\pi k_{\mathrm{w}}}\left(\frac{(1-\varphi)}{b^{2}}\right)+\frac{2 a q_{\mathrm{p}}}{\pi k_{\mathrm{w}}}\left(\frac{1}{a^{2}}+\frac{\varphi}{b^{2}-a^{2}}\right) \sum_{n=1}^{\infty} \\
& {\left[J_{1}\left(\frac{a}{b} \alpha_{n}\right) J_{0}\left(\frac{r}{b} \alpha_{n}\right) /\left(\alpha_{n}^{2} J_{0}^{2}\left(\alpha_{n}\right)\right)\right] \tanh \left(\frac{\alpha_{n}}{b} l_{\mathrm{w}}\right) }
\end{aligned}
$$

Thus, the average temperature of the droplet base could be obtained as:

$\bar{T}_{\mathrm{b}}=\frac{\int_{0}^{b} 2 \pi r T_{\mathrm{b}}(r) d r}{\pi b^{2}}$

$\bar{T}_{\mathrm{b}}=T_{w}+\frac{q_{p} l_{\mathrm{w}}}{\pi k_{\mathrm{w}}}\left(\frac{(1-\varphi)}{b^{2}}\right)$

The average temperature of the liquid-solid (droplet basemicropillar tip) interface at the droplet base could be estimated as:

$T_{\mathrm{b}, \mathrm{p}}=\frac{\int_{0}^{a} 2 \pi r T_{\mathrm{b}}(r) d r}{\pi a^{2}}$

$$
\begin{aligned}
& T_{\mathrm{b}, \mathrm{p}}=\bar{T}_{\mathrm{b}} \\
& \quad+\left\{\frac{2 a q_{\mathrm{p}}}{\pi k_{\mathrm{w}}}\left(\frac{1}{a^{2}}+\frac{\varphi}{b^{2}-a^{2}}\right) \sum_{n=1}^{\infty}\left[J_{1}^{2}\left(\frac{a}{b} \alpha_{n}\right) /\left(\alpha_{n}^{3} J_{0}^{2}\left(\alpha_{n}\right)\right)\right] \tanh \left(\frac{\alpha_{n}}{b} l_{\mathrm{w}}\right)\right\}
\end{aligned}
$$

Considering the heat conduction in the silicon substrate, the temperature on the tip of the micropillar could be calculated as:

$T_{\mathrm{p}}=T_{\mathrm{s}}-\frac{q_{\mathrm{p}}}{R_{\mathrm{s}}}$

The temperature at the liquid-solid (droplet base-micropillar tip) interface can be approximated by the contact temperature [39-41], so we have:

$T_{\mathrm{b}, \mathrm{p}}=T_{\mathrm{e}}=\frac{\sqrt{\rho_{\mathrm{w}} k_{\mathrm{w}} c_{\mathrm{w}}} T_{\mathrm{o}}+\sqrt{\rho_{\mathrm{Si}} k_{\mathrm{Si}} c_{\mathrm{Si}}} T_{\mathrm{p}}}{\sqrt{\rho_{\mathrm{w}} k_{\mathrm{w}} c_{\mathrm{w}}}+\sqrt{\rho_{\mathrm{Si}} k_{\mathrm{Si}} c_{\mathrm{Si}}}}$

where $\rho, c$ and $k$ are the density, specific heat and thermal conductivity of water (w) and silicon ( $\mathrm{Si}$ ), respectively; $T_{0}$ is the initial temperature or last iterative temperature (Section 3.5) of the water droplet.

As $\tanh \left(\frac{\alpha_{n}}{b} l_{\mathrm{w}}\right) \sim 1$, the average temperature of the water droplet base is given by:

$\bar{T}_{\mathrm{b}}=T_{\mathrm{e}}-\frac{2 a q_{p}}{\pi k_{w}}\left(\frac{1}{a^{2}}+\frac{\varphi}{b^{2}-a^{2}}\right) \sum_{n=1}^{\infty}\left[J_{1}^{2}\left(\frac{a}{b} \alpha_{n}\right) /\left(\alpha_{n}^{3} J_{0}^{2}\left(\alpha_{n}\right)\right)\right]$

\subsection{Evaporation from droplet cap surface}

For the sessile droplet evaporation at the relatively low temperature $\left(40^{\circ} \mathrm{C}-80^{\circ} \mathrm{C}\right)$, we only considered the conduction heat transfer in the bulk water and the internal convection process was neglected as discussed before. Thus, we employed one-dimensional heat conduction model to describe the heat transfer process inside the droplet as shown in Fig. 3(c). The water droplet was discretized into a series of thin layers $(i=1, \ldots X$, where $X$ is the total number of water layers) parallel to the substrate. Here, temperatures at the bottom surface $\left(T_{\mathrm{i}}\right)$ and the top surface $\left(T_{\mathrm{i}+1}\right)$ of the $i$ th water layer are assumed to be laterally uniform, respectively. Then, in the $i$ th water layer, we have:

$T_{\mathrm{i}+1}=T_{\mathrm{i}}-q_{\mathrm{w}, \mathrm{i}} \cdot R_{\mathrm{i}}$

where $R_{\mathrm{i}}=\frac{\Delta h}{k_{\mathrm{w}} \pi r_{\mathrm{i}}^{2}}$ is the thermal resistance of the $i$ th water layer, $\Delta h$ is the thickness of each water layer, and $r_{\mathrm{i}}$ is the radius of the $i$ th water layer.

Taking the $i$ th layer as the control volume, heat transfer into the $i$ th water layer from the bottom surface $\left(q_{\mathrm{w}, \mathrm{i}}\right)$ is balanced by the heat transfer to the top surface $\left(q_{\mathrm{w}, \mathrm{i}+1}\right)$ and the evaporation heat transfer rate $\left(q_{1, \mathrm{i}}\right)$ from the side surface. Thus, the energy balance in the $i$ th water layer is:

$q_{\mathrm{w}, \mathrm{i}+1}=q_{\mathrm{w}, \mathrm{i}}-q_{\mathrm{l}, \mathrm{i}}$

where $q_{1, \mathrm{i}}=J_{\mathrm{m}, \mathrm{i}}(r) \cdot h_{\mathrm{fg}} \cdot \Delta S_{\mathrm{i}}, \Delta S_{\mathrm{i}}$ is the side surface area of the $i$ th water layer, $h_{\mathrm{fg}}$ is the latent heat, and $J_{\mathrm{m}, \mathrm{i}}(r)$ is the local evaporation mass flux.

Then we applied the diffusion-driven model to estimate the local evaporation flux, which is the solution to the Laplace equation based on Fick's law of vapor diffusion around the droplet. Hence, the exact solution of the local evaporation mass flux is given by [19]:

$$
\begin{aligned}
J_{\mathrm{m}}(r)= & \frac{D_{\mathrm{d}}\left[c_{\mathrm{s}}\left(T_{\mathrm{i}}\right)-R_{\mathrm{h}} c_{\mathrm{s}}\left(T_{\infty}\right)\right]}{r_{c}} \cdot\left[\frac{1}{2} \sin \theta+\sqrt{2}(\cosh \beta+\cos \theta)^{3 / 2}\right. \\
& \left.\cdot \int_{0}^{\infty} \frac{\cosh (\theta \omega)}{\cosh (\pi \omega)} \tan [(\pi-\theta) \omega] \cdot P_{-0.5+i \omega}(\cosh \beta) \omega \cdot \mathrm{d} \omega\right]
\end{aligned}
$$

where $T_{\mathrm{i}}$ is approximated to be the temperature at the side surface, $c_{\mathrm{s}}$ is the saturated vapor concentration, $D_{\mathrm{d}}$ is the diffusion coefficient of vapor, $R_{\mathrm{h}}$ is the relative humidity, $\theta$ is the apparent contact angle of droplet, $\beta$ is the toroidal coordinate uniquely related to the cylindrical coordinate $r$ on the cap surface, $\omega$ is the variable of integration, and $P_{-0.5+i \omega}(x)$ is the Legendre function of the first kind.

The evaporation heat transfer rate from the droplet cap surface is calculated by integrating all the local evaporation heat transfer rates:

$q_{\mathrm{c}}=\sum_{i=1}^{X} q_{1, \mathrm{i}}$

The evaporation heat transfer rate from the droplet cap should be the same as the heat transferred through the water droplet:

$q_{\mathrm{c}}=(1-\varphi) q_{\mathrm{s}}$

As mentioned above, the temperature measured by the IR camera can be taken as the average temperature of the upper hemispherical surface as illustrated in Fig. 3(c). With the average droplet base temperature obtained, we can calculate the surface temperature distribution based on the heat transfer equation Eq. (20) for each of the discretized water layers. Thus, we can approximate the average temperature of the upper hemispherical surface of the droplet as:

$T_{\text {model }}=\sum_{i=1}^{M} T_{\mathrm{i}} \cdot \Delta S_{\mathrm{i}} / 2 \pi r_{w}^{2}$

where $M$ is the number of discrete water layers in the upper hemisphere of the droplet cap (here $M<X$ ), and $r_{\mathrm{w}}$ is the radius of the spherical cap.

\subsection{Algorithm for calculating the surface temperature and evaporation ratio}

The algorithm for calculating the droplet surface temperature and the evaporation ratio $\varphi$ is shown in the flowchart of Fig. 4. The 


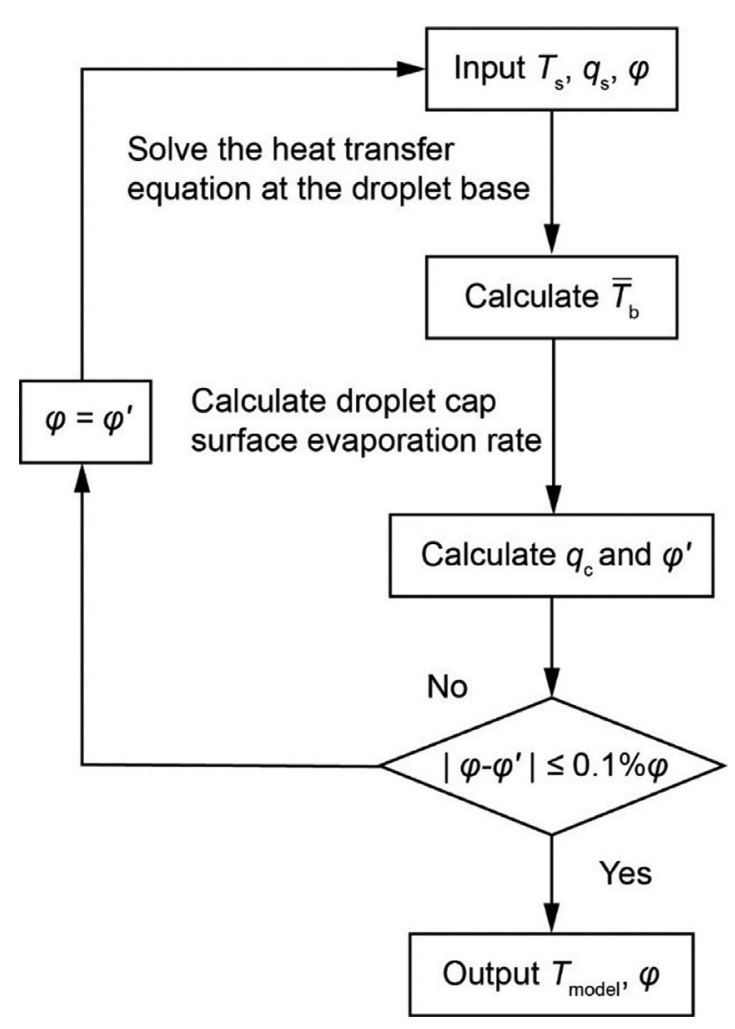

Fig. 4. Flowchart for calculating the droplet surface temperature and evaporation ratio $\varphi$, which is defined as the ratio of evaporation heat transfer rate from the droplet base surface to the total heat transfer rate through a droplet.

base temperature of the substrate $T_{S}$ is measured by the thermocouple and the heat transfer rate from the substrate $q_{s}$ is obtained by calculating the droplet volume decreasing rate based on Eq. (5). An initial estimate of the evaporation ratio $\varphi=0.5$ is used in the boundary condition to solve the heat transfer equation Eq. (9) at the droplet base. Then the temperature distribution at the droplet base is calculated by Eq. (14) and consequently the average temperature of the droplet base $\bar{T}_{b}$ is obtained. The nonuniform surface temperature distribution of the droplet cap is calculated based on the one-dimensional heat conduction model inside the droplet and the evaporation heat transfer rate $q_{\mathrm{c}}$ from the droplet cap is then obtained. The evaporation heat transfer rate from the droplet cap should be the same as the heat transfer through the droplet body from the droplet base and the value of $\varphi^{\prime}$ is then obtained by Eq. (24) as the new evaporation ratio in the next iteration of calculation. After the iteration loop achieves convergence, both the stable evaporation ratio $\varphi$ and the temperature distribution on the droplet cap surface are obtained. With the temperature distribution on the droplet cap obtained, the average temperature of the upper hemispherical surface of the droplet cap surface $T_{\text {model }}$ is calculated and compared with the experimental results for the validation of our thermal circuit model.

It is not practical to directly measure or calculate the evaporation heat transfer rate from the droplet base because of the complex micro-pillared structures on the substrate. Thus, the evaporation heat transfer rate from the droplet base is taken as the difference of the total evaporation heat transfer rate of the droplet and the evaporation heat transfer rate from the droplet cap. The evaporation heat transfer rate from the droplet cap is thus estimated by the diffusion-driven model with the droplet surface temperature gradient taken into consideration Eqs. 22 and (23). This evaporation heat transfer rate estimation method can be validated by Gleason and Putnam's work [42], in which they reported the droplet evaporation rate by the diffusion-driven model with the droplet surface temperature distribution taken into account and the estimation errors of this method were $1.84 \%$ and $2.83 \%$ for droplet evaporation on a substrate at $50^{\circ} \mathrm{C}$ and $65^{\circ} \mathrm{C}$, respectively. In this study, experimental measurements or observations of the droplet contact angle and contact radius evolutions are needed for the theoretical analysis and estimation of the evaporation heat transfer rate from the droplet cap surface and that from the droplet base surface.

\section{Results and discussion}

\subsection{Droplet evaporation dynamics}

The temporal evolutions of the droplet volume on the three sample substrates heated at different temperatures are shown in Fig. 5. In general, droplet volume decreases nonlinearly during the evaporation. The total evaporation time decreases with the rise of the substrate temperature. At the same substrate temperature, the total evaporation time increases with the increasing micropillar periodicity. It can be seen in Fig. 5 that at each substrate temperature, droplet evaporation has the longest total evaporation time on sample 3 (60 $\mu \mathrm{m}$ periodicity) and has the shortest total evaporation time on sample 1 ( $40 \mu \mathrm{m}$ periodicity). The observed increasing trend of total evaporation time can be ascribed to the enhanced thermal resistance between the droplet base and the substrate, which is caused by the increase of the micropillar periodicity with more air or vapor pockets. The total evaporation time of a $4 \mu \mathrm{L}$ water droplet on the three samples with varying base temperatures is shown in Table 2.

The evolutions of droplet contact angle and nondimensional contact radius with nondimensional evaporation time are shown in Fig. 6. Here the nondimensional contact radius is defined as the ratio of the contact radius to the initial contact radius, and the nondimensional time is defined as the ratio of the evaporation time to the total evaporation time. It can be seen from Fig. 6 that the evolutions of droplet contact angle and nondimensional contact radius are almost the same for droplet evaporation on each sample with different substrate temperatures. For droplet evaporation on sample 1 (Fig. 6a), the droplet contact angle continuously decreased during the first $68 \%$ portion of the total evaporation time while the contact radius remained unchanged, which was in the constant contact radius (CCR) mode during droplet evaporation. After the contact angle reached the receding contact angle, the contact angle stopped decreasing and was maintained at a constant level while the contact radius started to decrease, which was in the constant contact angle (CCA) mode of droplet evaporation. The droplet evaporation was kept in the CCA mode till the nondimensional time approached 0.9. Then, both the contact angle and contact radius started decreasing, which was the mixed mode for droplet evaporation. For droplet evaporation on the same

Table 2

Total evaporation time for a water droplet of $4 \mu \mathrm{L}$ on micro-pillared substrates with different base temperatures.

\begin{tabular}{lll}
\hline Substrate & Substrate base temperature $\left({ }^{\circ} \mathrm{C}\right)$ & Total evaporation time $(\mathrm{s})$ \\
\hline \multirow{3}{*}{ Sample 1} & 40 & $620.1 \pm 13.7$ \\
& 60 & $293.3 \pm 9.6$ \\
& 80 & $156.8 \pm 10.6$ \\
\hline \multirow{2}{*}{ Sample 2 } & 40 & $627.1 \pm 7.6$ \\
& 60 & $312.2 \pm 6.5$ \\
& 80 & $177.1 \pm 10.6$ \\
\hline \multirow{3}{*}{ Sample 3 } & 40 & $633.3 \pm 10.2$ \\
& 60 & $322.3 \pm 7.7$ \\
& 80 & $184.1 \pm 12.9$ \\
\hline
\end{tabular}




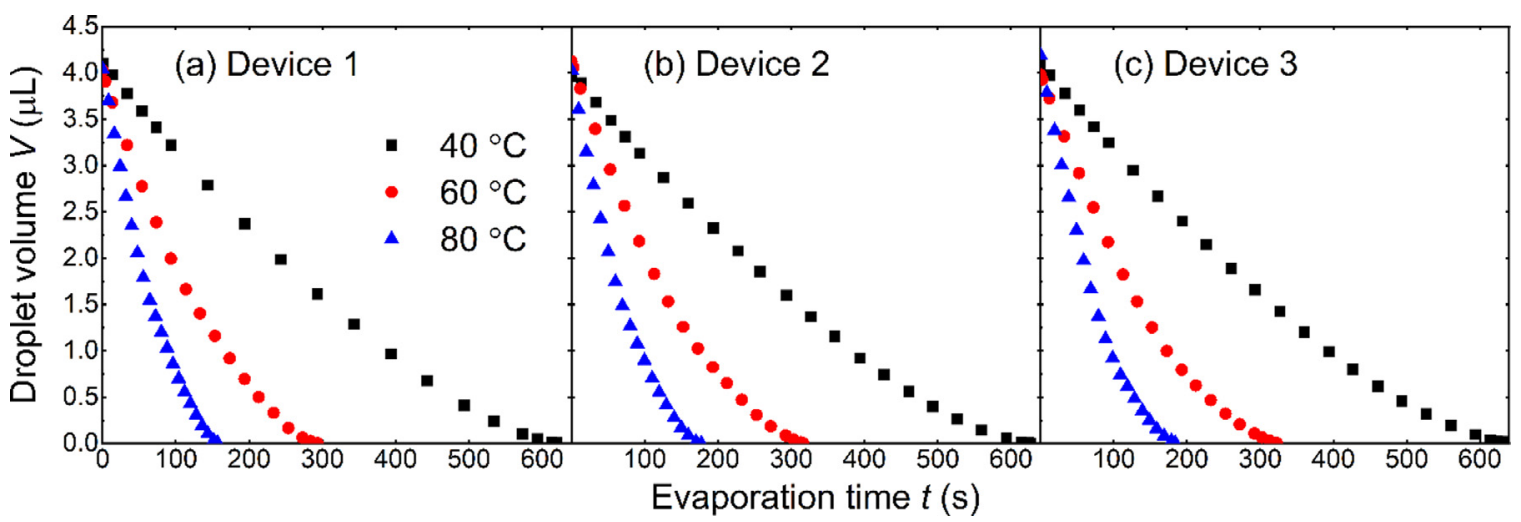

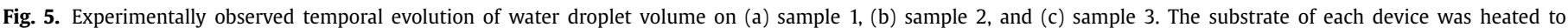
$40^{\circ} \mathrm{C}, 60^{\circ} \mathrm{C}$ and $80^{\circ} \mathrm{C}$, respectively. The initial droplet volume was $4 \mu \mathrm{L}$.

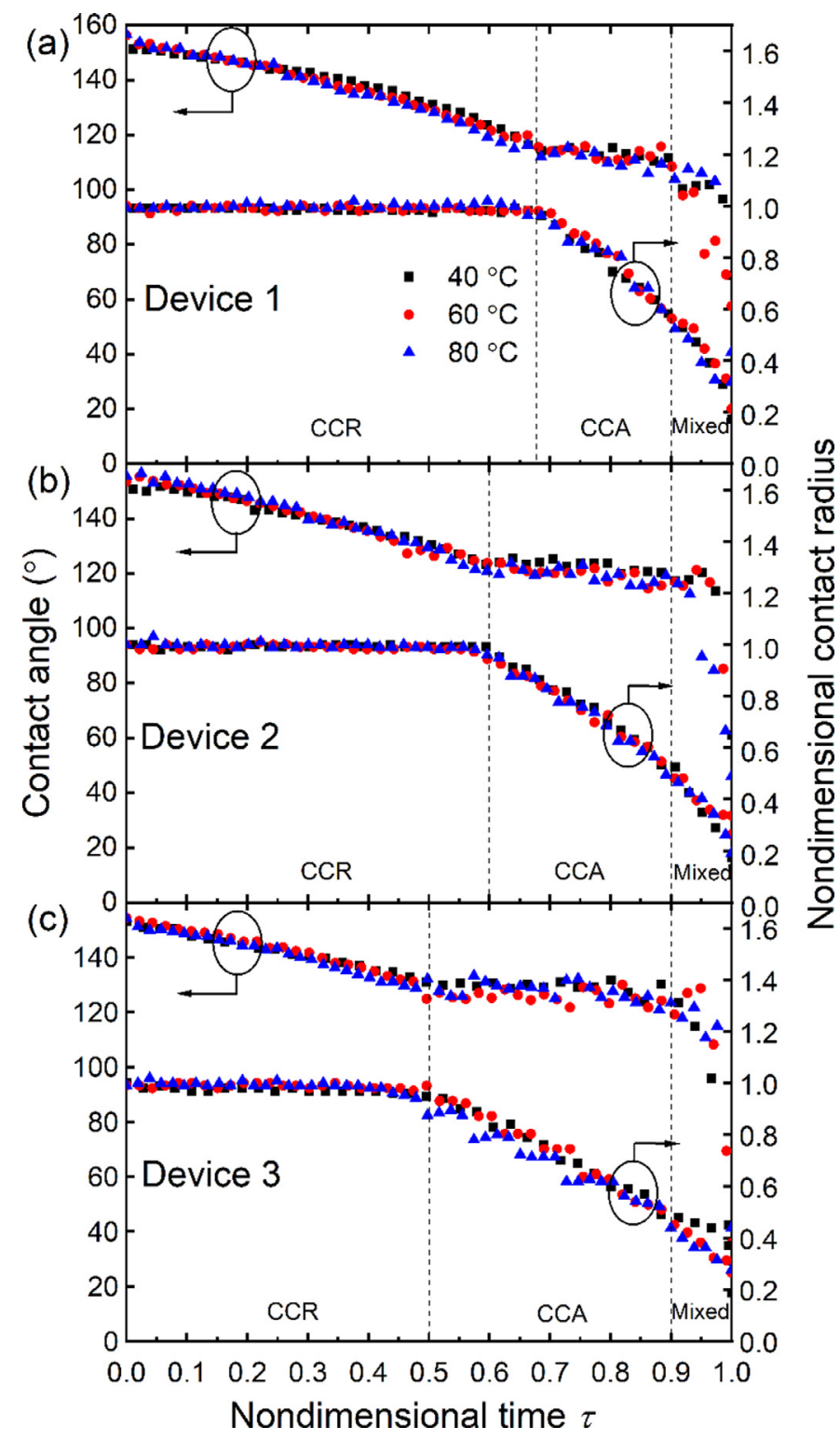

Fig. 6. Experimental evolution of droplet contact angle and nondimensional contact radius versus nondimensional time on (a) sample 1, (b) sample 2 and (c) sample 3. Each substrate base was heated to $40^{\circ} \mathrm{C}, 60^{\circ} \mathrm{C}$ and $80^{\circ} \mathrm{C}$, respectively. sample with different substrate temperatures, the evaporation process had almost the same compositions of the CCR, CCA and mixed modes, indicating that the substrate structure is more influential on the evaporation mode transition than the substrate temperature at least within a certain temperature range (i.e., $\leq 80^{\circ} \mathrm{C}$ ). The evolutions of droplet contact angle and nondimensional contact radius on sample 2 (Fig. 6b) and sample 3 (Fig. 6c) are like that on sample 1 . It is noteworthy that the receding contact angle increases with the increasing periodicity of micropillars on the substrates. The receding contact angles on sample 1, sample 2 and sample 3 are about $112^{\circ}, 120^{\circ}$ and $128^{\circ}$, respectively. Since the initial contact angles of water droplets on these three devices are almost the same (about $155^{\circ}$ ), a larger receding contact angle results in the shorter term of the CCR mode. The nondimensional evaporation times for the CCR mode on sample 1, sample 2 and sample 3 are about $0.68,0.6$ and 0.5 , respectively.

\subsection{Experimental and modelled droplet cap surface temperature}

In this work, IR camera was used to monitor the droplet cap temperature evolution with droplet evaporation. The snapshots of IR thermography for water droplet evaporation on sample 2 heated at $40^{\circ} \mathrm{C}, 60^{\circ} \mathrm{C}$ and $120^{\circ} \mathrm{C}$, respectively, are shown in Fig. 7. Distinct surface temperature gradient exhibits in these IR images. The average temperature of the droplet cap surface was extracted from these IR images for comparison with that predicted by the thermal circuit model. As such, the effects of the droplet surface curvature on IR thermography are discussed in the supplementary materials.

The evolutions of droplet cap temperature versus the nondimensional time on samples 1,2 and 3 are shown in Figs. 8(a), (b) and (c), respectively. For droplet evaporation on substrates with temperature in the range of $22^{\circ} \mathrm{C}-80^{\circ} \mathrm{C}$, the droplet cap temperature predicted by the thermal circuit model matches well with that measured by the IR camera. There exists a large temperature gradient between the droplet cap surface and the substrate base, which is caused by the thermal resistances of the droplet body, the evaporation at the droplet surface and the conduction through the silicon substrate. It can be seen in Fig. 8 that the average droplet cap temperature increased in the CCR mode and kept almost constant in the CCA mode. Similar trends were also observed by Saenz et al. in their numerical simulation of droplet evaporation on heated surfaces [43]. During the CCR mode, the thermal resistance of the droplet body decreases with the decreasing droplet volume. Meanwhile, the continuously shrinking droplet cap gets closer to the substrate and thus the droplet cap temperature increases. During the CCA mode, the contact area between the droplet base and the substrate continuously decreases. As a result, less heat is transferred into the droplet, hindering the temperature increase of the 
(a)

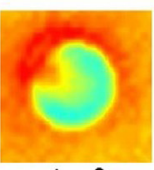

(b)

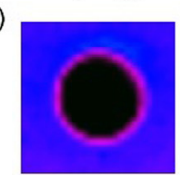

$t=0 s$

(c)

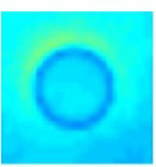

$t=0 s$

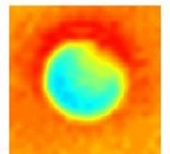

$t=70 s$

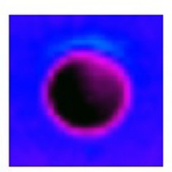

$t=34 \mathrm{~s}$

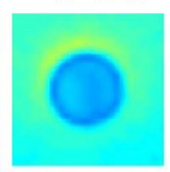

$t=4.2 \mathrm{~s}$

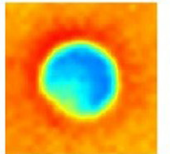

$t=140 s$

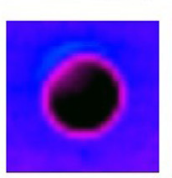

$t=68 \mathrm{~s}$

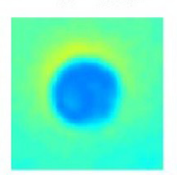

$t=8.4 \mathrm{~s}$

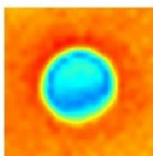

$t=210 s$

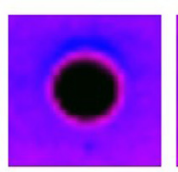

$t=102 s$

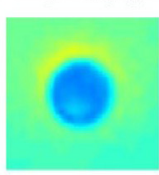

$t=12.6 s$

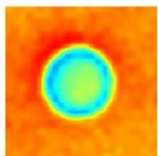

$t=280 s$

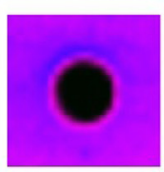

$t=136 s$

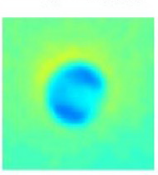

$t=16.8 s$

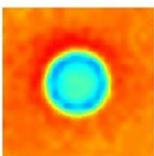

$t=350 s$

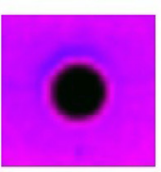

$t=170 s$

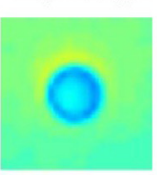

$t=21.0 s$
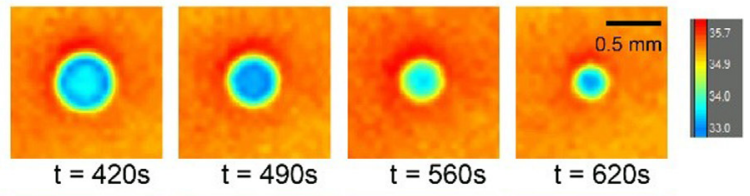

$t=490 s$

$\mathrm{t}=560 \mathrm{~s}$

$t=620 s$

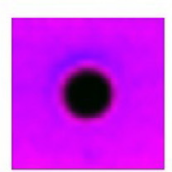

$t=204 s$

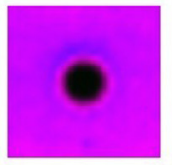

$t=238 \mathrm{~s}$

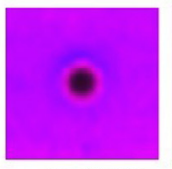

$t=272 s$

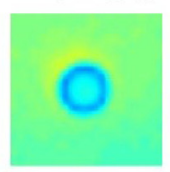

$t=25.2 s$

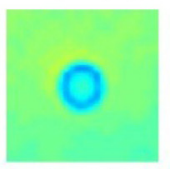

$t=29.4 \mathrm{~s}$

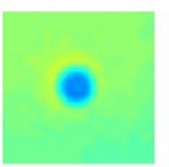

$t=33.6 s$

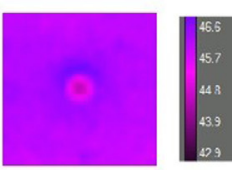

$t=306 s$

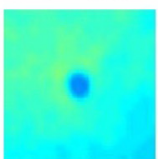

$t=37.8 \mathrm{~s}$

Fig. 7. Snapshots of IR thermography of droplet evaporation on sample 2 heated at (a) $40^{\circ} \mathrm{C}$, (b) $60^{\circ} \mathrm{C}$ and (c) $120^{\circ} \mathrm{C}$, respectively. The initial droplet volume was $4 \mu \mathrm{L}$.
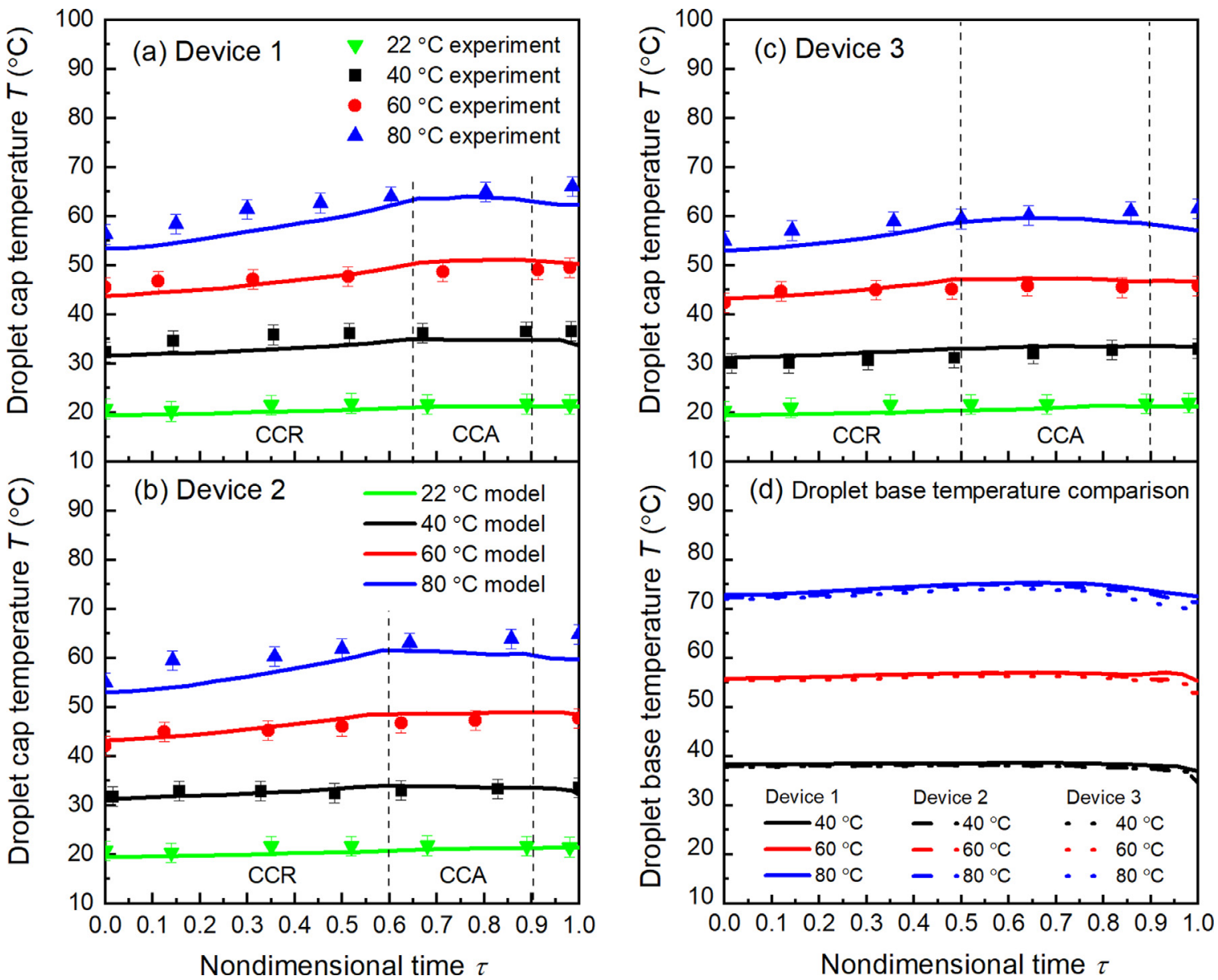

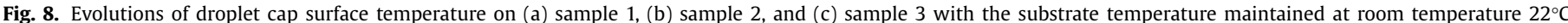

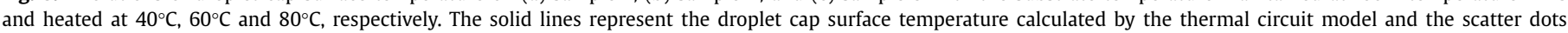

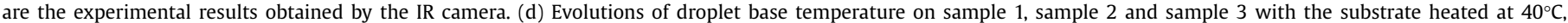
$60^{\circ} \mathrm{C}$ and $80^{\circ} \mathrm{C}$, respectively.

droplet. During the CCA mode, the shrinking contact area between the droplet base and the substrate results in the almost unchanged temperature on the droplet cap. In the mixed mode, the volume of the evaporating droplet is relatively small $(<0.05 \mu \mathrm{L})$ and droplet evaporation in this mode is not a focus of this discussion.

The average temperature of the droplet base on each sample with different substrate temperatures is shown in Fig. 8(d). There exists an apparent temperature mismatch between the droplet base and the substrate base. Though there is a temperature drop through the substrate because of the substrate's conduction resistance, the large thermal conductivity of silicon $\left(300 \mathrm{~W} \cdot \mathrm{m}^{-1} \cdot \mathrm{K}^{-1}\right)$ should have led to a very small thermal resistance. As such, the temperature drop through the substrate itself is estimated to be $<1^{\circ} \mathrm{C}$. However, the temperature mismatches between the droplet 

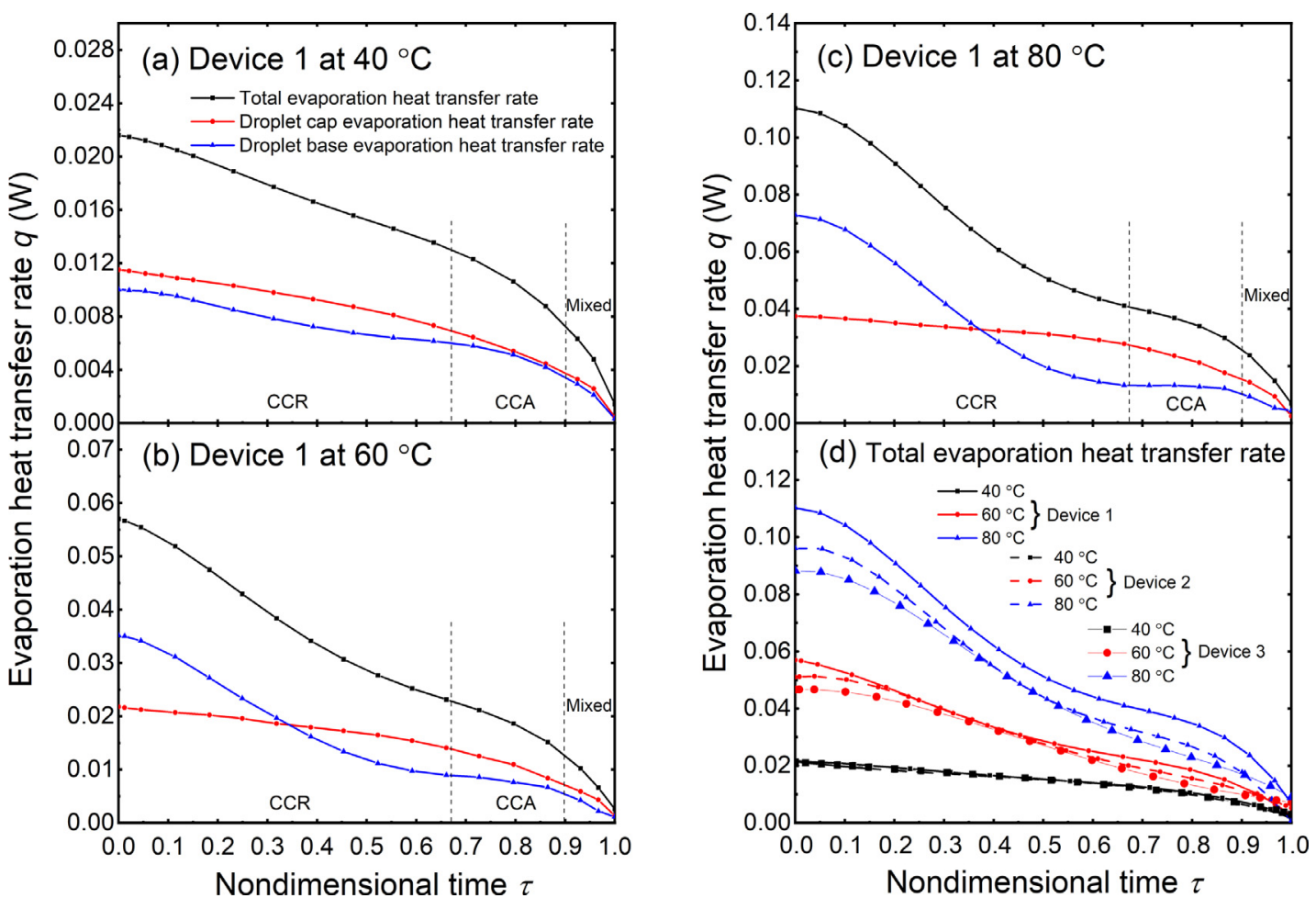

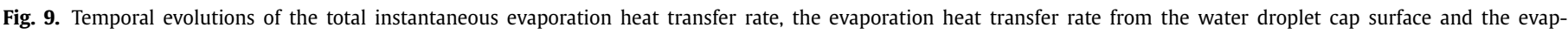

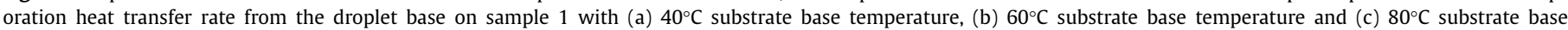

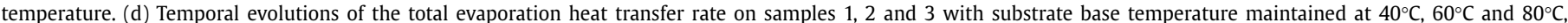
respectively. The initial droplet volume was $4 \mu \mathrm{L}$.

base and the substrate base are about $2^{\circ} \mathrm{C}, 5^{\circ} \mathrm{C}$ and $8^{\circ} \mathrm{C}$ with the substrate base temperature maintained at $40^{\circ} \mathrm{C}, 60^{\circ} \mathrm{C}$ and $80^{\circ} \mathrm{C}$, respectively. It is plausible that such an apparent temperature difference between the droplet base and the substrate base may be mainly caused by the local evaporation from the droplet base within the interstitial spaces between the micropillars.

In Figs. 9(a), (b) and (c), we show the temporal evolutions of the evaporation heat transfer rate from the droplet cap surface, the evaporation heat transfer rate from the droplet base, and the summation of the above two heat transfer rates, i.e., the total instantaneous evaporation heat transfer rate, on sample 1 with the substrate base temperature maintained at $40^{\circ} \mathrm{C}, 60^{\circ} \mathrm{C}$ and $80^{\circ} \mathrm{C}$, respectively. The corresponding evaporation heat transfer rates of water droplets on sample 2 and sample 3 are given in the supplementary materials. The total instantaneous evaporation heat transfer rate of a sessile water droplet is calculated by Eqs. (5) and (6) in term of the droplet volume decreasing rate. The evaporation heat transfer rate from the droplet cap surface is calculated by the diffusiondriven model with the cap surface temperature distribution predicted by the thermal circuit model. The evaporation heat transfer rate from the droplet base is the difference between the total evaporation heat transfer rate and the evaporation heat transfer rate from the droplet cap. Obviously, the increase of the substrate temperature would lead to the evaporation enhancement from both the droplet cap surface and the droplet base surface. Yet, both the evaporation rate from the droplet cap and that from the droplet base decrease during droplet evaporation on a substrate with a constant temperature. In the CCR mode the slope of the evaporation rate from the droplet cap is smaller than that at the droplet base, indicating a lower decreasing speed of the evaporation rate from the droplet cap. As reflected by Eq. (22), the reduction of the droplet contact angle in the CCR mode results in the decrease of the evaporation rate from the droplet cap surface. Meanwhile, the temperature increase on the droplet cap surface will enhance the evaporation rate thereon. The effect of droplet cap temperature increase compensates the depressed effect of the droplet contact angle decrease on the evaporation from the droplet cap. The combined effects of the contact angle decrease and the droplet cap temperature increase give rise to the slow decrease of the evaporation rate from the droplet cap in the CCR mode. In the CCA mode, the average temperature of the droplet cap keeps essentially constant, which is different from the increasing trend of the droplet cap temperature in the CCR mode. Meanwhile, the contact radius of the droplet decreases in the CCA mode, which causes the decreasing heat transfer area between the droplet and the substrate. Thus, the evaporation rate from the droplet cap decreases quickly in the CCA mode. In summary, in the CCR mode the decrease of the total evaporation rate of the droplet is mainly caused by the decreasing evaporation rate from the droplet base. Whereas in the CCA mode, the decrease of the total evaporation rate is mainly induced by the decreasing evaporation rate from the droplet cap.

Droplet evaporation on different substates exhibits distinct evaporation heat transfer rates as shown in Fig. 9(d). Droplet on sample 1 (40 $\mu \mathrm{m}$ periodicity) has the maximum evaporation heat transfer rate while droplet has the minimum evaporation rate on device 3 (60 $\mu \mathrm{m}$ periodicity). Due to the different periodicities of micropillars, the droplet-micropillar contact areas are different on these devices. Since water droplets on these sample substrates have almost the same apparent contact area (i.e., base area of the droplet), small periodicity of micropillars means larger heat transfer area between the droplet base and the substrate, i.e., the solidliquid interface area. The larger solid-liquid interface area results in the higher droplet evaporation rate at the same substrate temperature.

Evaporation ratio $\varphi(\tau)$ is defined as the ratio of the evaporation heat transfer rate from the droplet base to the total evapo- 


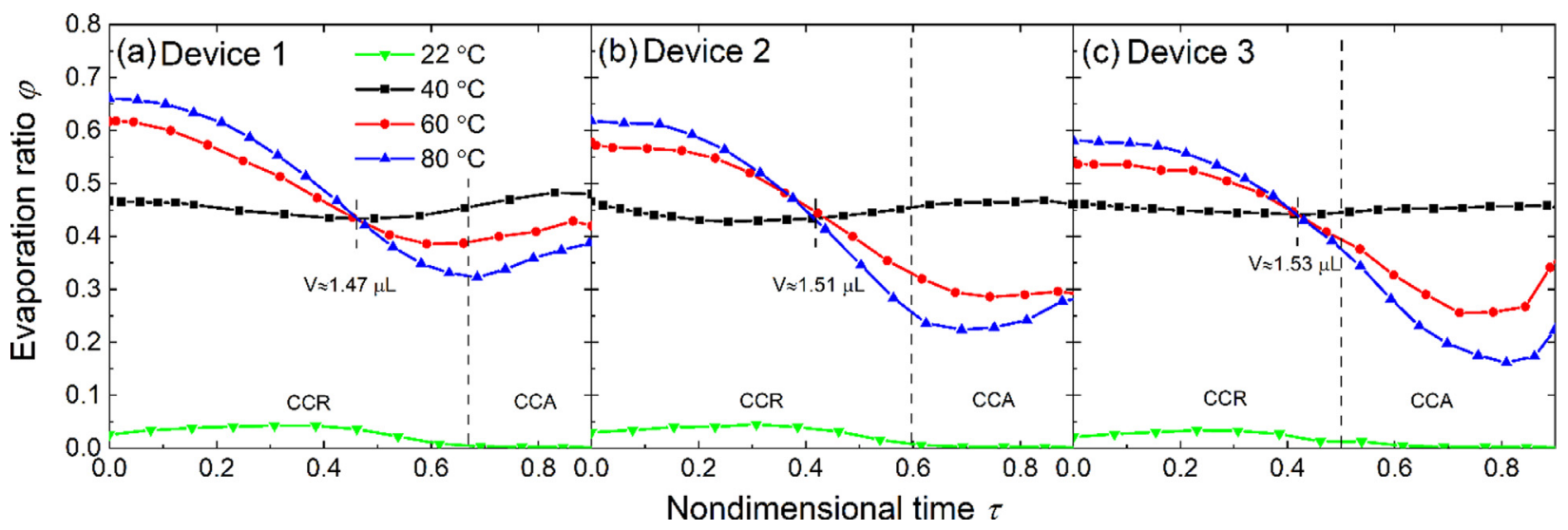

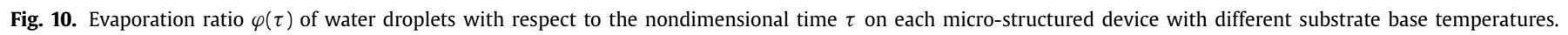

ration heat transfer rate at each moment $\tau$. The evaporation ratio $\varphi$ with respect to nondimensional time $\tau$ on different sample devices with different substrate base temperatures is shown in Fig. 10. The evaporation ratio $\varphi$ generally decreases in the CCR mode while increasing close to the end of the CCA mode. This phenomenon is mainly caused by the relatively larger decreasing rate of evaporation from the droplet base in the CCR mode and the relatively smaller decreasing rate of evaporation in the CCA mode, compared with the evaporation rate from the droplet cap. Generally, the evaporation ratio $\varphi$ increases with the substrate temperature for droplet with a relatively large volume. For droplet evaporation at room temperature, the evaporation ratio $\varphi$ is almost 0 , which means most of the evaporation occurs at the droplet cap surface. The increase of the substrate temperature will directly cause a temperature increase at the droplet base and subsequently induce the temperature increase at the droplet cap. Due to the thermal resistance of the droplet bulk, the temperature increase at the droplet cap is smaller than that at the droplet base in response to the substrate temperature increase. Thus, more evaporation occurs from the droplet base than that from the droplet cap with the increase of the substrate temperature. It can be seen from Fig. 10 that the evaporation ratio $\varphi$ decreases with the increase of the substrate temperature after the droplet volume shrank to less than $1.5 \mu \mathrm{L}$. This opposite variation of the evaporation ratio is caused by the decreased droplet volume. When the droplet volume shrank to about $1.5 \mu \mathrm{L}$, both the droplet height and the droplet cap surface area decreased rapidly, e.g., the surface area of the droplet cap could be reduced by half. As a result, the thermal resistance of the droplet bulk is comparatively small, and the increase of the substrate temperature will cause a higher temperature rise at the droplet cap. Thus, stronger evaporation occurs from the droplet cap than from the droplet base with the increase of the substrate temperature and consequently the evaporation ratio $\varphi$ decreases.

\subsection{Effective conductivity of water droplet for evaporation on high temperature substrates}

We further conducted droplet evaporation experiments on the same micro-structured surfaces at even higher temperatures. Figs. 11(a), (b) and (c) show the evolutions of droplet cap temperature during droplet evaporation on different samples with the substrate temperature maintained at $100^{\circ} \mathrm{C}$ and $120^{\circ} \mathrm{C}$, respectively. The dash lines are the droplet cap temperatures calculated by the thermal circuit model. For droplet evaporation on a substrate with a relatively low temperature $\left(40^{\circ} \mathrm{C}-80^{\circ} \mathrm{C}\right)$, the droplet cap temperature calculated by the thermal circuit model matches well with the experimental results. However, for droplet evaporation on a substrate with a relatively high temperature $>100^{\circ} \mathrm{C}$, there exist large deviations between the model-predicted results and the experimental measurements. These deviations are largely due to the internal fluid flow of the droplet, which becomes even stronger at higher temperatures and is not considered in the thermal circuit model. The effects of droplet internal flow are not significant for droplet evaporation on relatively low temperature substates $\left(40^{\circ} \mathrm{C}\right.$ - $80^{\circ} \mathrm{C}$ ). As mentioned by Dash and Garimella [23], the characteristic velocity of fluid flow in a $3 \mu \mathrm{L}$ water droplet evaporating on a $40^{\circ} \mathrm{C}-60^{\circ} \mathrm{C}$ substrate is of tens of microns per second and the Peclet number is less than 1 . Thus, it is reasonable for us to only consider the conduction heat transfer while neglecting the internal convection of the water droplet on a substrate in the moderate temperature range of $40^{\circ} \mathrm{C}-80^{\circ} \mathrm{C}$. With the further increase of substrate temperature, more heat is transferred through the droplet and the temperature difference between the droplet top and base becomes larger. Fluid motion driven by the temperature gradient in the droplet becomes violent and the characteristic velocity of internal flow increases. Thus, we need to account for the effects of the droplet internal flow in the thermal circuit model for high temperature evaporation analysis.

Driven by the surface tension gradient (caused by the surface temperature gradient), water at the droplet bottom will flow upwards, which will in turn diminish the temperature gradient on the droplet surface. As a result, the upper hemispherical part of the droplet will get warmed up by the up-flowing hot water, and the temperature in the upper hemispherical portion should become relatively higher than the case without internal flow. To account for the effect of internal fluid flow, the overall averaged droplet cap surface temperature is calculated as the modified droplet cap temperature:

$T_{\text {model }}^{\prime}=\sum_{i=1}^{X} T_{\mathrm{i}} \cdot \Delta S_{\mathrm{i}} / 2 \pi r_{w}^{2}$

As shown in Figs. 11(a), (b) and (c), the modified temperature calculated by Eq. (26) matches well with the experimental data for droplet evaporation on samples 1, 2 and 3 with the substrate base temperature maintained at $100^{\circ} \mathrm{C}$. But there still exist large deviations between the modified results and the experimental data for droplet evaporation on sample devices with the substrate temperature heated up to $120^{\circ} \mathrm{C}$. In this condition, the convection heat transfer in the droplet cannot be neglected. In the thermal circuit model, only the conductive thermal resistance of the droplet is considered. Convection inside the droplet will remarkably enhance heat transfer therein and reduce the total thermal resistances of the droplet. Because of the complexity of internal flow, it is not practical to analyze the convection heat transfer simply based on the droplet characteristic velocity. Alternatively, an effective ther- 

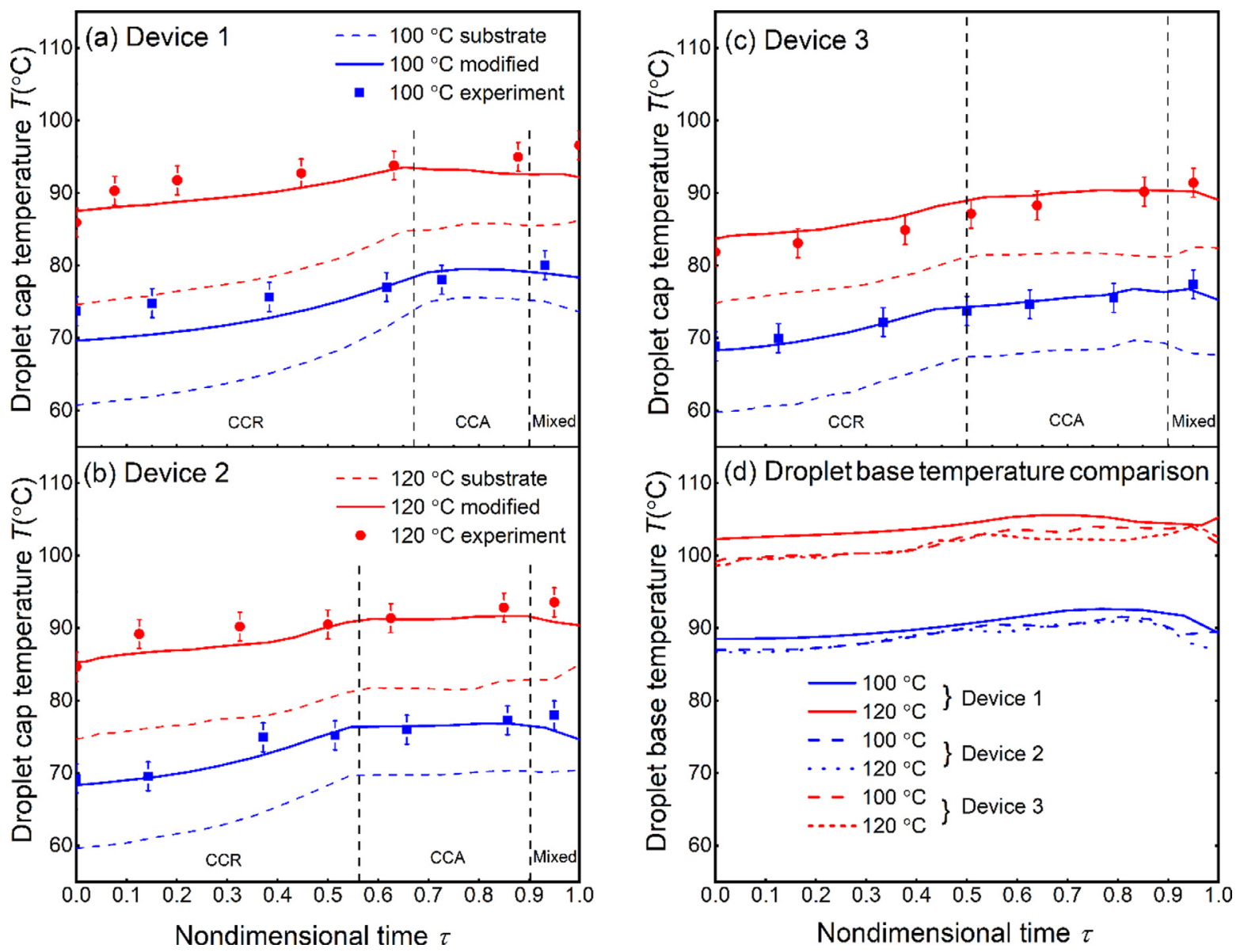

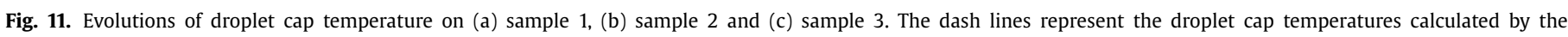

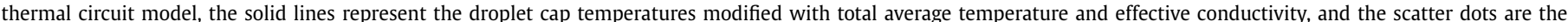

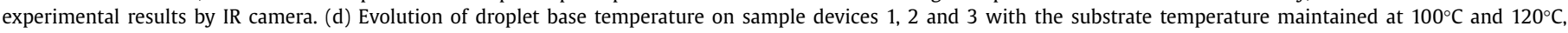
respectively.

mal conductivity $k_{\text {eff,w }}$ is adopted by us to account for both the convection and conduction heat transfer in the droplet. As such, the effective conductivity [44] of an evaporating droplet is defined as:

$k_{\text {eff,w }}=e \cdot k_{w}$

where $e$ is a coefficient of the effective conductivity. The effective conductivity $k_{\mathrm{eff}, \mathrm{w}}$ takes place of the original water conductivity $k_{w}$ in the thermal circuit model to account for both the conduction and the convection heat transfer in the droplet. In this scenario, the effect of convection in heat transfer is approximately equivalent to an increase of the thermal conductivity of the working fluid. The minimum value of $e$ allowing for the average difference between the model predicted surface temperature and the experimental value less than $2^{\circ} \mathrm{C}$, i.e., $\left|\bar{T}_{\text {model }}-\bar{T}_{\text {expt }}\right| \leq 2^{\circ} \mathrm{C}$, is chosen as the coefficient of the effective conductivity.

The coefficient $e$ for the effective thermal conductivity of droplet evaporation on samples 1, 2 and 3 with the substrate temperature maintained at $120^{\circ} \mathrm{C}$ was found to be $3.2,2.9$ and 2.7 , respectively. Our estimates of coefficient $e$ are very close to the numerically fitted coefficient of 2.72 that was reported by Abramzon and Sirignano [44]. In our experiments, these sample substrates had been heated to a level over the boiling temperature of water. However, no boiling was observed in the droplet even with the substrate temperature reaching $120^{\circ} \mathrm{C}$, which is possibly due to the evaporative cooling at the droplet base. As evaporation at the droplet base could cool down the local surface temperature, we calculated the average temperature of the droplet base as shown in Fig. 11(d). For droplet evaporation on the $100^{\circ} \mathrm{C}$ substrate, the base temperature was about $88^{\circ} \mathrm{C}$ and for droplet evaporation on the $120^{\circ} \mathrm{C}$ substrate, the base temperature was about $102^{\circ} \mathrm{C}$, which is very close to the saturation temperature of $100^{\circ} \mathrm{C}$ at the ambient condition. Our analysis shows that evaporative cooling from the droplet base can significantly delay the onset of droplet boiling with the substrate temperature even reaching $120^{\circ} \mathrm{C}$.

\section{Conclusions}

In this work, we experimentally and theoretically investigated the evaporation of water droplet on hot micro-pillared superhydrophobic surfaces. Only few studies investigated the sessile droplet evaporation on substrates within the relative low temperature range of $40^{\circ} \mathrm{C}-100^{\circ} \mathrm{C}$ and very limited attention had been paid to the local evaporation from the droplet base. For the first time, the evaporation heat transfer rate at the droplet base from the interstitial liquid-vapor interface between micropillars was carefully evaluated by us. According to our analysis, droplet cap temperature shows distinct trends in different evaporation modes, i.e., droplet cap temperature increases in the CCR mode while keeping almost constant in the CCA mode. The total evaporation rate from both the droplet cap and base generally decreases during the evaporation. In the CCA mode, the decrease of the total droplet evaporation rate is largely induced by the decreasing evaporation rate from the droplet cap. In the CCR mode, however, the decrease of the total evaporation rate is mainly caused by the decreasing evaporation rate from the droplet base. The evapora- 
tion ratio $\varphi$ decreases in the CCR mode and increases approaching the end of the CCA mode. Also, the evaporation ratio increases with the increasing substrate temperature during the early stage of evaporation when the droplet volume still remains comparatively large. After the droplet shrinks to a small volume, the evaporation ratio decreases with the increasing substrate temperature.

Internal fluid motion and convection start playing a more influential role on droplet evaporation on substrates with a relatively high temperature $\left(100^{\circ} \mathrm{C}-120^{\circ} \mathrm{C}\right)$ and an effective thermal conductivity $k_{\text {eff,w }}$, which is about three times of water conductivity $k_{w}$, was employed by us as a correction factor for the thermal circuit model to account for the convection heat transfer in the water droplet. Furthermore, the average temperature at the droplet base was estimated and a large temperature difference between the droplet base and the substrate surface was unveiled. The apparent temperature differences between the droplet base and the substrate base were about $2^{\circ} \mathrm{C}, 5^{\circ} \mathrm{C}, 8^{\circ} \mathrm{C}, 13^{\circ} \mathrm{C}$ and $18^{\circ} \mathrm{C}$ for the evaporating droplet on the micro-structured substrates with their base temperature maintained at $40^{\circ} \mathrm{C}, 60^{\circ} \mathrm{C}, 80^{\circ} \mathrm{C}, 100^{\circ} \mathrm{C}$ and $120^{\circ} \mathrm{C}$, respectively. Our study about the sessile droplet evaporation on hot micro-structured surfaces reveals the relative importance of heat and mass transfer from the droplet base and the evaporative cooling thereon. Moreover, the thermal circuit model developed by us provides a convenient tool to analyze the evaporation dynamics of a sessile droplet on hot micro-structured substrates with a broad range of surface temperatures.

\section{Declaration of Competing Interest}

None.

\section{CRediT authorship contribution statement}

Wenge Huang: Conceptualization, Methodology, Investigation, Formal analysis, Writing - original draft. Xukun He: Conceptualization, Methodology, Writing - review \& editing. Yahua Liu: Supervision, Funding acquisition. Jiangtao Cheng: Conceptualization, Supervision, Project administration, Funding acquisition, Writing review \& editing.

\section{Acknowledgment}

This work is financially supported by NSF CBET under grant 1550299, NSF ECCS under grant 1808931, NSF CBET under grant 2133017 and NSFC under grant 52075071. Partial fabrication of silicon micropillars was conducted at the Center for Nanophase Materials Sciences, which is a DOE Office of Science User Facility.

\section{Supplementary materials}

Supplementary material associated with this article can be found, in the online version, at doi:10.1016/j.ijheatmasstransfer. 2021.122314.

\section{References}

[1] P. Calvert, Inkjet Printing for Materials and Devices, Chem. Mater. 13 (10) (2001) 3299-3305.

[2] A. Wu, L. Yu, Z. Li, H. Yang, E. Wang, Atomic force microscope investigation of large-circle DNA molecules, Anal. Biochem. 325 (2) (2004) 293-300.

[3] W. Jia, H.H. Qiu, Experimental investigation of droplet dynamics and heat transfer in spray cooling, Exp. Therm. Fluid Sci. 27 (7) (2003) 829-838.

[4] J. Song, W. Cheng, M. Nie, X. He, W. Nam, J. Cheng, W. Zhou, Partial Leidenfrost Evaporation-Assisted Ultrasensitive Surface-Enhanced Raman Spectroscopy in a Janus Water Droplet on Hierarchical Plasmonic Micro-/Nanostructures, ACS Nano 14 (8) (2020) 9521-9531.

[5] X. He, J. Cheng, Evaporation-Triggered Directional Transport of Asymmetrically Confined Droplets, J. Colloid Interface Sci. 604 (2021) 550-561.
[6] S.A. Putnam, A.M. Briones, L.W. Byrd, J.S. Ervin, M.S. Hanchak, A. White, J.G. Jones, Microdroplet evaporation on superheated surfaces, Int. J. Heat Mass Transf. 55 (21) (2012) 5793-5807.

[7] P. Tsai, R.G. Lammertink, M. Wessling, D. Lohse, Evaporation-triggered wetting transition for water droplets upon hydrophobic microstructures, Phys. Rev. Lett. 104 (11) (2010) 116102.

[8] L. Zhao, J. Cheng, The mechanism and universal scaling law of the contact line friction for the Cassie-state droplets on nanostructured ultrahydrophobic surfaces, Nanoscale 10 (14) (2018) 6426-6436.

[9] L.S. Lam, M. Hodes, R. Enright, Analysis of Galinstan-Based Microgap Cooling Enhancement Using Structured Surfaces, J. Heat. Transfer 137 (9) (2015).

[10] A. Al-Sharafi, B.S. Yilbas, H. Ali, Droplet Heat Transfer on Micropost Arrays With Hydrophobic and Hydrophilic Characteristics, J. Heat. Transfer 140 (7) (2018).

[11] L. Bansal, S. Chakraborty, S. Basu, Confinement-induced alterations in the evaporation dynamics of sessile droplets, Soft Matter 13 (5) (2017) 969-977.

[12] S. Semenov, F. Carle, M. Medale, D. Brutin, Boundary conditions for a one-sided numerical model of evaporative instabilities in sessile drops of ethanol on heated substrates, Phys. Rev. E 96 (6-1) (2017) 063113.

[13] P.a. Bexon, The Evaporation of Sessile or Pendant Drops in Still Air, J. Colloid Interface Sci. 61 (2) (1977) 336-350.

[14] X.K. He, J.T. Cheng, C.P. Collier, B.R. Srijanto, D.P. Briggs, Evaporation of squeezed water droplets between two parallel hydrophobic/superhydrophobic surfaces, J. Colloid Interface Sci. 576 (2020) 127-138.

[15] M.E.R. Shanahan, Simple Theory of "Stick-Slip" Wetting Hysteresis, Langmuir 11 (3) (1995) 1041-1043.

[16] N.N. Lebedev, Special Functions and Their Applications, Prentice-Hall, Englewood Cliffs, NJ, USA, 1965.

[17] R.G.L. Hua Hu, Evaporation of a Sessile droplet on a substrate, J. Phys. Chem. B 106 (2002) 1334-1344.

[18] Robert D. Deegan, Olgica Bakajin, Todd F. Dupont, Greg Huber, Sidney R. Nagel, T.A. Witten, Contact line deposits in an evaporating drop, Phys. Rev. E 62 (2000).

[19] Y.O. Popov, Evaporative deposition patterns: spatial dimensions of the deposit, Phys. Rev. E 71 (3) (2005) 1-17.

[20] M.A. Kadhim, N. Kapur, J.L. Summers, H. Thompson, Experimental and Theoretical Investigation of Droplet Evaporation on Heated Hydrophilic and Hydrophobic Surfaces, Langmuir 35 (19) (2019) 6256-6266.

[21] T.A.H. Nguyen, A.V. Nguyen, M.A. Hampton, Z.P. Xu, L. Huang, V. Rudolph, Theoretical and experimental analysis of droplet evaporation on solid surfaces, Chem. Eng. Sci 69 (1) (2012) 522-529.

[22] H. Gelderblom, Á.G. Marín, H. Nair, A. van Houselt, L. Lefferts, J.H. Snoeijer, D. Lohse, How water droplets evaporate on a superhydrophobic substrate, Phys. Rev. E 83 (2) (2011) 026306.

[23] S. Dash, S.V. Garimella, Droplet evaporation on heated hydrophobic and superhydrophobic surfaces, Phys. Rev. E 89 (2014) 042402.

[24] S. Dash, S.V. Garimella, Droplet evaporation dynamics on a superhydrophobic surface with negligible hysteresis, Langmuir 29 (34) (2013) 10785-10795.

[25] A. Aldhaleai, F. Khan, T. Thundat, P.A. Tsai, Evaporation dynamics of water droplets on superhydrophobic nanograss surfaces, Int. J. Heat Mass Transf. 160 (2020).

[26] A. Bussonnière, M.B. Bigdeli, D.-Y, Chueh, Q Liu, P. Chen, P.A. Tsai, Universal wetting transition of an evaporating water droplet on hydrophobic micro- and nano-structures, Soft Matter 13 (5) (2017) 978-984.

[27] K. Gleason, H. Voota, S.A. Putnam, Steady-state droplet evaporation: Contact angle influence on the evaporation efficiency, Int. J. Heat Mass Transf. 101 (2016) 418-426.

[28] R.N. Wenzel, Resistance of Solid Surfaces to Wetting by Water, Ind. \& Eng. Chem. 28 (8) (1936) 988-994.

[29] G. McHale, S. Aqil, N.J. Shirtcliffe, M.I. Newton, H.Y. Erbil, Analysis of droplet evaporation on a superhydrophobic surface, Langmuir 21 (2005) 11053-11060.

[30] S. Adera, R. Raj, R. Enright, E.N. Wang, Non-wetting droplets on hot superhydrophilic surfaces, Nat. Commun. 4 (2013) 2518.

[31] M. Wei, Y. Song, Y. Zhu, D.J. Preston, C.S. Tan, E.N. Wang, Heat transfer suppression by suspended droplets on microstructured surfaces, Appl. Phys. Lett. 116 (23) (2020).

[32] H.-m. Kwon, J.C. Bird, K.K. Varanasi, Increasing Leidenfrost point using micronano hierarchical surface structures, Appl. Phys. Lett. 103 (20) (2013).

[33] M. Badv, I.H. Jaffer, J.I. Weitz, T.F. Didar, An omniphobic lubricant-infused coating produced by chemical vapor deposition of hydrophobic organosilanes attenuates clotting on catheter surfaces, Sci. Rep. 7 (1) (2017) 11639.

[34] R. Hays, D. Maynes, J. Crockett, Thermal transport to droplets on heated superhydrophobic substrates, Int. J. Heat Mass Transfer 98 (2016) 70-80.

[35] Y. Maeda, F. Lv, P. Zhang, Y. Takata, D. Orejon, Condensate droplet size distribution and heat transfer on hierarchical slippery lubricant infused porous surfaces, Appl. Therm. Eng. 176 (2020) 115386.

[36] T.L. Bergman, F.P. Incropera, D.P. DeWitt, A.S. Lavine, Fundamentals of heat and mass transfer, John Wiley \& Sons, 2011.

[37] Seri Lee, Seaho Song, K.P.Moran Van Au, Constriction Spreading resistance model for electroics packing, ASME/JSME Thermal Engineering Conference, 1995.

[38] D.P. Kennedy, Spreading Resistance in Cylindrical Semiconductor Devices, Int. J. Appl. Phys. 31 (8) (1960) 1490-1497.

[39] M. di Marzo, D.D. Evans, Evaporation of a water droplet deposited on a hot high thermal conductivity solid surface, United States, 1986. 
[40] K.M. Itaru Michiyoshe, Heat transfer characteristics of evaporation of a liquid droplet on heated surfaces international, Int. J. Heat Mass Transf. 21 (1977) 605-613.

[41] M. Seki, H. Kawamura, K. Sanokawa, Transient temperature profile of a hot wall due to an impinging liquid droplet, J. of Heat Transf. 100 (1987) 167-169.

[42] K. Gleason, S.A. Putnam, Microdroplet evaporation with a forced pinned contact line, Langmuir 30 (34) (2014) 10548-10555.
[43] P.J. Sáenz, K. Sefiane, J. Kim, O.K. Matar, P. Valluri, Evaporation of sessile drops: a three-dimensional approach, J. Fluid Mech. 772 (2015) 705-739.

[44] W.A.S.B. Abrambon, Droplet vaporization model for spray combusition calculations, Int. J. Heat Mass Transf. 32 (1989) 1605-1618. 